$9-2006$

\title{
Obtaining Mode Mixity for a Bimaterial Interface Crack Using the Virtual Crack Closure Technique
}

\author{
A. Agrawal \\ University of Delaware
}

Anette M. Karlsson

Cleveland State University, a.karlsson@csuohio.edu

Follow this and additional works at: https://engagedscholarship.csuohio.edu/enme_facpub

Part of the Mechanical Engineering Commons

How does access to this work benefit you? Let us know!

Publisher's Statement

The final publication is available at Springer via http://dx.doi.org/10.1007/s10704-006-0069-4

\section{Original Citation}

Agrawal, A., and Karlsson, A. M., 2006, "Obtaining Mode Mixity for a Bimaterial Interface Crack using the Virtual Crack Closure Technique," International Journal of Fracture, 141(1-2) pp. 75-98.

This Article is brought to you for free and open access by the Mechanical Engineering Department at EngagedScholarship@CSU. It has been accepted for inclusion in Mechanical Engineering Faculty Publications by an authorized administrator of EngagedScholarship@CSU. For more information, please contact library.es@csuohio.edu. 


\title{
Obtaining mode mixity for a bimaterial interface crack using the virtual crack closure technique
}

\author{
A. Agrawal • A. M. Karlsson
}

\begin{abstract}
We review, unify and extend work pertaining to evaluating mode mixity of interfacial fracture utilizing the virtual crack closure technique (VCCT). From the VCCT, components of the strain energy release rate (SERR) are obtained using the forces and displacements near the crack tip corresponding to the opening and sliding contributions. Unfortunately, these components depend on the crack extension size, $\Delta$, used in the VCCT. It follows that a mode mixity based upon these components also will depend on the crack extension size. However, the components of the strain energy release rate can be used for determining the complex stress intensity factors (SIFs) and the associated mode mixity. In this study, we show that several-seemingly different-suggested methods presented in the literature used to obtain mode mixity based on the stress intensity factors are indeed identical. We also present an alternative, simpler quadratic equation to this end. Moreover, a $\Delta$-independent strain energy release based mode mixity can be defined by introducing a "normalizing length parameter." We show that when the reference length (used for the SIF-based mode mixity) and the normalizing length (used for $\Delta$ independent SERR-based mode mixity) are equal,
\end{abstract}

\footnotetext{
A. Agrawal · A. M. Karlsson ( $ه)$

Department of Mechanical Engineering, University of

Delaware, 126 Spencer Laboratory, Newark, 19716,

DE, USA

e-mail: karlsson@udel.edu
}

the two mode mixities are only shifted by a phase angle, depending on the bimaterial parameter $\varepsilon$.

Keywords Interface crack - Complex stress intensity factor - Virtual crack closure technique . SIF-based mode mixity - SERR-based mode mixity

\section{Introduction}

Bimaterial interfaces are intrinsic in many engineering applications, ranging from microelectronics to adhesive joints, from fiber-reinforced composites to thermal barrier coatings. These layered structures undergo complex failure modes, many times relating in interfacial cracking (e.g., Wang and Suo 1990; Karlsson and Evans 2001). Thus, to design reliable layered structures, it is paramount that the mechanics of the interface crack is understood. Fracture toughness of bimaterial interfaces has received attention through analytical (e.g., Rice 1988; Hutchinson and Suo 1992), experimental (e.g., Charalambides et al. 1989; Wang and Suo 1990; Yuuki et al. 1994; Ikeda et al. 1998) and numerical simulations (e.g., Sun and Jih 1987; Matos et al. 1989; Toya 1992; Beuth 1996; Bjerken and Persson 2001).

Contrary to homogeneous, isotropic materialswhere cracks tend to propagate in pure mode I locally at the crack tip-mode mixity is a critical 
parameter for interfacial fractures. The mode mixity (sometimes called the phase angle of fracture) is the relative proportions of tractions ahead of the crack tip in sliding mode (mode II) and opening mode (mode I) in the facture. A crack constrained in an interface is subjected to mixed mode conditions - and propagates in mixed mode-when the preferred fracture path is in the interface. There is ample experimental evidence that interfacial fracture toughness depends strongly on the mode mixity (e.g., Evans et al. 1990; Wang and Suo 1990; O'Dowd et al. 1992; Yuuki et al. 1994). Typically, the total fracture toughness increases as the mode II contribution increases.

Mode mixity can be determined using a stress intensity factor (SIF)-based approach or can be based on the components of the strain energy release rate (SERRs). Although analytical expressions for the total strain energy release rate and SIF-based mode mixity are available for some simple interfacial crack problems (Hutchinson and Suo 1992; Cherepanov 1979), they involve considerable mathematical complexity. For complicated geometries or loading conditions, the analytical expressions may not be available. Thus, numerical methods are many times preferred. The total strain energy release rate can be obtained through numerical computation of the J-integral (Rice 1968) using finite element based techniques (e.g. the virtual crack extension technique, Parks 1974). However, the J-integral provides no information about the mode mixity.

Stress-based mode mixity can be determined by two alternative numerical methods:

(i) The crack-face displacement method (Matos et al. 1989). The method is widely used and is easy to implement for most problems. It requires the displacement field for many nodes close to the crack tip, and may be difficult to implement if the crack faces are not straight.

(ii) The M-Integral method (Yau et al. 1980). The method requires a known auxiliary solution and is very sensitive to the accuracy of the auxiliary solution because the error builds up in a quadratic manner.
A third method, the virtual crack closure technique (VCCT), has successfully been used to obtain both the total strain energy release rate and the mode mixity for cracks in homogeneous materials (Rybicki and Kanninen 1977; Dattaguru et al. 1994, Xie et al. 2004). For an interface crack, the VCCT has traditionally been used to obtain the total strain energy release rate. Obtaining mode mixity for an interface crack using the VCCT has proven to be more challenging (Sun and Jih 1987; Raju et al. 1988; Dattaguru et al. 1994). However, several approaches have been suggested to extract consistent mode mixity values using the VCCT (Toya 1992; Chow and Atluri 1995; Beuth 1996; Sun and Qian 1997; Bjerken and Persson 2001).

The purpose of this paper is to present the VCCT as a reliable and efficient method to extract SIF- or SERR-based mode mixity to characterize an interface crack, within the context of linear-elastic fracture mechanics. To this end, we will in Chapters 3-4 re-derive the equations that are needed to extract mode mixity from the VCCT results of SERRs using asymptotic stress and displacement field near the crack tip. The derivation is intended to establish a clear link between various approaches (Toya 1992; Chow and Atluri 1995; Beuth 1996; Sun and Qian 1997). Furthermore, a simple quadratic equation is derived that can be used to obtain the SIFbased mode mixity. This equation gives identical results to the previous approaches suggested by Toya (1992), Chow and Atluri (1995) and Sun and Qian (1997). In addition, an approach by Bjerken and Persson (2001) is examined and found attractive for providing acceptable values of mode mixity with significantly less computational efforts. Further, a modified definition of mode mixity, based on SERRs (Beuth 1996), is shown as an alternative measure for characterizing an interface crack.

In Chapter 5, we will numerically illustrate the theory discussed in Chapters 2-4, with two benchmark problems: an interface crack in an infinite bimaterial plate subjected to uniform normal stress and a bi-layer four-point flexure specimen with an interface crack. We believe that reading the numerical examples parallel with the theory may help a reader who is unfamiliar with the concepts presented herein. 


\section{The interface crack problem}

In this section, we will review the definitions and some key concepts relating to the complex stress intensity factor, the SIF-based mode mixity, the total strain energy release rate, and end with a discussion relating to the contact zone.

\subsection{Nature of the interface crack singularity}

A bimaterial body experiences stress intensification from both a geometric discontinuity (crack), as well as a material discontinuity. Stress intensification for a plane interface crack between two elastic, isotropic materials was first suggested by Westergaard (1939). A complete analytical solution was given by Williams (1959), where the asymptotic nature of the dominant singular stress field using an eigenfunction approach was established. That work indicated that the stress field is coupled near the crack tip and that the stress singularity is of the order of $r^{\xi}$, where $\xi=-1 / 2+i \varepsilon$ is the complex eigenvalue, and $\varepsilon$ is the bimaterial constant defined as:

$\varepsilon=\frac{1}{2 \pi} \ln \left[\frac{1-\beta}{1+\beta}\right]$

where

$\alpha=\frac{\overline{E_{1}}-\overline{E_{2}}}{\overline{E_{1}}+\overline{E_{2}}}, \quad$ and $\quad \beta=\frac{\mu_{1}\left(\vartheta_{2}-1\right)-\mu_{2}\left(\vartheta_{1}-1\right)}{\mu_{1}\left(\vartheta_{2}+1\right)+\mu_{2}\left(\vartheta_{1}+1\right)}$.

$\alpha, \beta$ are the "Dundur's parameters" (Dudurs 1969); and $j=1,2$ represents material 1 and 2 (Fig. 1), respectively. Furthermore, $\vartheta_{j}=\left(3-4 v_{j}\right)$ and $\overline{E_{j}}=$ $E_{j} /\left(1-v_{j}^{2}\right)$ for plane strain; and $\vartheta_{j}=\left(3-v_{j}\right) /$ $\left(1+v_{j}\right)$ and $\bar{E}_{j}=E_{j}$ for plane stress. $E_{j}$ is the elastic modulus, $v_{j}$ is Poisson's ratio and $\mu_{j}=E_{j} / 2\left(1+v_{j}\right)$ is the shear modulus. The oscillatory singularity is given by

$r^{i \varepsilon}=\cos (\varepsilon \ln r)+i \sin (\varepsilon \ln r)$,

where $i=\sqrt{-1}$. An interface crack experiences mixed mode condition even when subjected to pure mode I loading.

The interface crack solutions based on the "open crack model" (e.g., Williams 1959; Rice and Sih

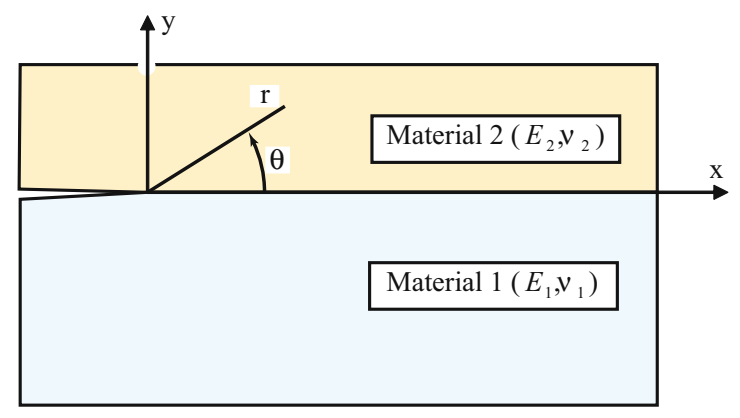

Fig. 1 Geometry and nomenclature for an interface crack

1965; Malyshev and Salganik 1965; England 1965) contain oscillation of stresses and displacements in the vicinity of the crack tip. In such model, the interpenetration of crack faces always occurs near the crack tip, which is physically unfeasible. Comninou (1977) presented a modified solution considering contact near the crack tip to eliminate the oscillations. However, the contact region is very small. Thus, the oscillatory solution allowing interpenetration is considered valid in the $K$-annulus, i.e. the region close to the crack tip where the singular field dominates, outside the non-linear contact zone (Rice 1988; Rice et al. 1990; Wang and Suo 1990).

\subsection{Definition of complex K and SIF-based mode mixity}

Using the convention for an interface crack defined in Fig. 1, the complex stress intensity factor, $K$, is given by (Rice and Sih 1965)

$K=K_{1}+i K_{2}=|K| e^{i \Psi^{*}}$,

where $K$ has units of $\mathrm{Nm}^{-2} \sqrt{\mathrm{m}} \mathrm{m}^{-i \varepsilon}$ and $\Psi^{*}$ is the "phase angle" or "mode mixity" of $K$. In the $K$ annulus region, the tractions at a distance $r$ ahead of the crack tip at the interface $(\theta=0)$ are given by (Rice and Sih 1965; Rice 1988)

$\sigma_{y y}+i \sigma_{x y}=\frac{K}{\sqrt{2 \pi r}} r^{i \varepsilon}$.

An alternative definition of the interfacial stress intensity factors was suggested by Rice (1988), referred to as the complex stress intensity factors of classical type, which agrees with the definition of Malyshev and Salganik (1965). The stress intensity 
factors of classical type, $K_{\mathrm{I}}$ and $K_{\mathrm{II}}$, represent stress intensity factors of two different modes of fracture and can be defined as:

$K_{\mathrm{I}}+i K_{\mathrm{II}}=K l^{i \varepsilon}=|K| e^{i \psi}$,

where $l$ is an arbitrarily chosen reference length, $\psi$ is the mode mixity of $K l^{i \varepsilon}$, and $K_{\mathrm{I}}, K_{\mathrm{II}}$ are based on the reference length $r=l$. The stress intensity factors defined by Eq. 6 have the units of the "isotropic" stress intensity factors, i.e. $\mathrm{Nm}^{-2} \sqrt{\mathrm{m}}$, and are thus easy to interpret physically. However, the stress intensity factors $K_{\mathrm{I}}, K_{\mathrm{II}}$ for a bimaterial system with $\beta \neq 0$ are not directly analogous to mode I and mode II stress intensity factors for homogeneous material, since a characterizing reference length always needs to be specified (Rice 1988; Hutchinson and Suo 1992). The stress field in Eq. 5 can be rewritten in terms of $K_{\mathrm{I}}, K_{\mathrm{II}}$ as

$\sigma_{y y}+i \sigma_{x y}=\frac{K_{\mathrm{I}}+i K_{\mathrm{II}}}{\sqrt{2 \pi r}}\left(\frac{r}{l}\right)^{i \varepsilon}$.

The (stress-based) mode mixity in Eq. 6 is expressed as

$\psi=\tan ^{-1}\left(\frac{\operatorname{Im}\left[K r^{i \varepsilon}\right]}{\operatorname{Re}\left[K r^{i \varepsilon}\right]}\right)_{r=l}=\tan ^{-1}\left(\frac{K_{\mathrm{II}}}{K_{\mathrm{I}}}\right)$,

which is equivalent to

$\psi=\tan ^{-1}\left(\frac{\sigma_{x y}}{\sigma_{y y}}\right)_{r=l}$.

$\psi$ is the mode mixity of $K l^{i \varepsilon}$ and can be related to the mode mixity of $K$ as (Rice 1988; Hutchinson and Suo 1992; Ikeda et al. 1998)

$\psi=\Psi^{*}+\varepsilon \ln (l)$.

Futhermore,

$\psi_{2}=\psi_{1}+\varepsilon \ln \frac{l_{2}}{l_{1}}$

where $l_{1}$ and $l_{2}$ are two reference lengths used to define the mode mixities: $\psi_{1}=\psi_{1}\left(l_{1}\right)$ and $\psi_{2}=$ $\psi_{2}\left(l_{2}\right)$.

The expression for the displacement jumps is (Hutchinson and Suo 1992)

$$
\begin{aligned}
& \delta_{y}+i \delta_{x}=8 \frac{K_{\mathrm{I}}+i K_{\mathrm{II}}}{(1+2 i \varepsilon) E^{*} \cosh (\pi \varepsilon) \sqrt{2 \pi}} \sqrt{r}\left(\frac{r}{l}\right)^{i \varepsilon}, \\
& \text { where } \frac{1}{E^{*}}=\frac{1}{2}\left(\frac{1}{\overline{E_{1}}}+\frac{1}{\overline{E_{2}}}\right) .
\end{aligned}
$$

Here, $\delta_{x}$ and $\delta_{y}$ are the displacement jumps between two points located on opposite crack faces at a distance $r$ behind the crack tip, along $x$ - and $y$-directions, respectively. Lastly, the angle $\phi$ at a distance $r$ behind the crack tip is defined as

$\phi=\tan ^{-1}\left(\frac{\delta_{x}}{\delta_{y}}\right)$.

\subsection{Total strain energy release rate}

The total strain energy release rate can be expressed in terms of the modulus of complex $K$ as (Malyshev and Salganik 1965)

$G=\frac{1-\beta^{2}}{E^{*}}\left(|K|^{2}\right)$,

where $|K|^{2}=\left(K_{1}^{2}+K_{2}^{2}\right)=\left(K_{\mathrm{I}}^{2}+K_{\mathrm{II}}^{2}\right)$ and it is noted that $1-\beta^{2}=1 / \cosh ^{2}(\pi \varepsilon)$ and $\left|l^{i \varepsilon}\right|=1$. The strain energy release rate for an interface crack has the dimension of $\mathrm{Nm}^{-1}$ (which is the same as that for the strain energy release rate for monolithic material).

\subsection{Estimate of the contact zone}

For a bimaterial interface crack $(\beta \neq 0)$, the open crack solution with oscillatory stress and displacement field given by Eqs. 7 and 10, respectively, indicates that there are infinite numbers of sign changes of the normal and shear stress, and the normal and shear displacement, near the crack tip (Sun and Qian 1997). The oscillation zone for stress and displacement are not necessarily the same. Similarly, the oscillation zone based on the oscillatory field model and the contact zone based the contact model are not necessarily the same. Rice (1988) arrived at an elementary estimate of the contact zone as the radius of oscillation zone of displacement, $r_{\mathrm{o}}$, which is the largest value of $r$ for which the opening gap $\delta_{y}$ vanishes in the cycle of oscillation:

$\operatorname{Re}\left\{\frac{K_{\mathrm{I}}+i K_{\mathrm{II}}}{1+2 i \varepsilon}\left(\frac{l}{r_{\mathrm{O}}}\right)^{i \varepsilon}\right\}=0$

which gives

$r_{\mathrm{O}}=l \exp \left\{\frac{1}{\varepsilon} \tan ^{-1}\left(\frac{K_{\mathrm{I}}-2 \varepsilon K_{\mathrm{II}}}{K_{\mathrm{II}}+2 \varepsilon K_{\mathrm{I}}}\right)\right\}$. 
The contact zone obtained above is very small compared to the crack size and other specimen dimension even for large mismatch $(\beta=0.5)$. Indeed, the contact zone is much smaller than even the atomic dimensions for moderate values of $\beta$ (Rice 1988; Wang and Suo 1990; Sun and Qian 1997; Borovkov et al. 2000).

\section{Strain energy release rates for an interface crack}

In this section, we will introduce the virtual crack closure technique (VCCT) that is used to obtain the components of the strain energy release rate $G_{I}$ and $G_{\mathrm{II}}$. Due to the oscillatory nature of stress and displacement fields, the components of the strain energy release rates (SEERs) for an interface crack are oscillatory. Consequently, $G_{\mathrm{I}}$ and $G_{\mathrm{II}}$ become dependent on the assumed crack-extension size, $\Delta$, in the finite element simulations. Various procedures have been suggested to extract meaningful $\Delta$-independent parameters from the oscillatory SERRs in order to characterize the interface crack (Toya 1992; Chow and Atluri 1995; Beuth 1996; Sun and Qian 1997). We will show that the expressions for $G_{\mathrm{I}}$ and $G_{\mathrm{II}}$ used by these authors are identical. We will conclude with comments on the implications of $\Delta$-dependent quantities and introduce a coupled strain energy quantity.

\subsection{The virtual crack closure technique (VCCT)}

According to Irwin (1957), the work required to extend a crack by an infinitesimal distance is equal to the work required to close the crack to its original length. Thus, for homogeneous, isotropic, linearelastic material, the components of strain energy release rate for mode I and mode II can be expressed as:

$$
\begin{aligned}
& G_{\mathrm{I}}=\operatorname{Lim}_{\Delta \rightarrow 0} \frac{1}{2 \Delta} \int_{0}^{\Delta} \sigma_{y y}(\Delta-r) \delta_{y}(r) \mathrm{d} r, \\
& G_{\mathrm{II}}=\operatorname{Lim}_{\Delta \rightarrow 0} \frac{1}{2 \Delta} \int_{0}^{\Delta} \sigma_{x y}(\Delta-r) \delta_{x}(r) \mathrm{d} r,
\end{aligned}
$$

where $\Delta$ is a small crack extension; $\sigma_{y y}$ and $\sigma_{x y}$ are the normal and shear tractions, respectively, at a distance $r$ ahead of the crack tip; and $\delta_{x}$ and $\delta_{y}$ are the displacement jumps at a distance $r$ behind the crack tip, along the $x$ (sliding mode) and $y$ (opening mode) directions, respectively.

Rybicki and Kanninen (1977) showed that the integrals in Eqs. 15 and 16 can be computed numerically by finite element analysis using a technique referred to as the virtual crack closure technique (VCCT). This method can be used with conventional (non-singular), linear, finite element simulation to get accurate strain energy release rate values.

The VCCT is based on nodal forces and displacements near the crack tip. In this technique, the four elements adjacent to the tip should preferably have the same size (Fig. 2), where the length of the crack tip element size is $\Delta$. Nodes $e$ and $f$ are joined at the crack tip, whereas nodes $c$ and $d$ are next behind the crack tip on the two opposite crack faces. $F_{y, c}$ and $F_{x, c}$ are the forces required to hold nodes $c$ and $d$ together in the $y$ and $x$ directions, respectively. If the forces $F_{y, c}$ and $F_{x, c}$ are applied at nodes $c$ and $d$, the crack closes by increment $\Delta$, and $c$ and $d$ become coincident with each other to form the new crack tip. Thus, assuming that $\Delta$ is small compared to the overall length of the crack, $F_{y, c}$ and $F_{x, c}$ can be assumed to be the same as the tractions at the current crack tip (nodes $e$ and $f$ ). The forces at the nodes $e$ and $f$ can be obtained directly from the finite element results. Conversely, if we apply these forces at $e$ and $f$ to balance the crack

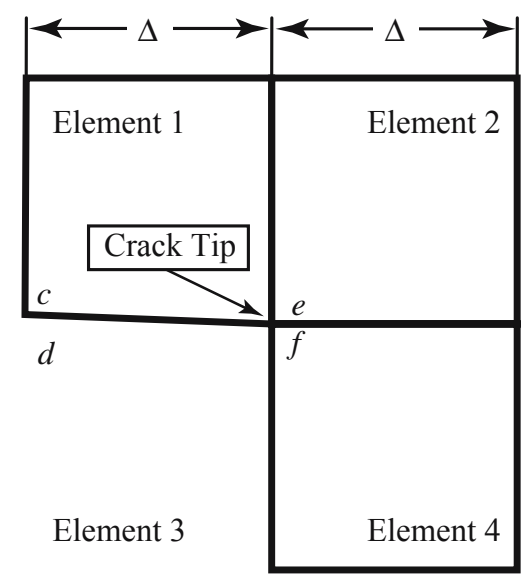

Fig. 2 Finite element mesh near the crack tip and nomenclature used in the virtual crack closure technique 
tip tractions, the crack will advance by $\Delta$ and the work done for the small unit crack extension corresponds to the (total) strain energy release rate.

Hence, recasting Eqs. 15 and 16 to capture the discrete nature of finite element analysis, the VCCT yields

$G_{\mathrm{I}}=\frac{1}{2 \Delta} F_{y, c} \delta_{y, c-d}$,

$G_{\mathrm{II}}=\frac{1}{2 \Delta} F_{x, c} \delta_{x, c-d}$,

where $\delta_{y, c-d}$ and $\delta_{x, c-d}$ are the relative opening and sliding displacement jumps between points $c$ and $d$, respectively. The components of the strain energy release rate for a crack can be obtained from Eqs. 17 and 18 using the VCCT. How this is done, is demonstrated in Sect. 5.1.1 (infinite plate) and Sect. 5.2.1 (4-point bending).

However, the procedures of isotropic fracture are not directly applicable to analyze bimaterial interfacial cracks. $G_{\mathrm{I}}$ and $G_{\mathrm{II}}$ depend on the crack extension size, do not converge as $\Delta$ approaches infinitesimal value, and do not relate to mode I and II mode contributions (Sun and Jih 1987; Raju et al. 1988; Dattaguru et al. 1994). Thus, $G_{\mathrm{I}}$ and $G_{\mathrm{II}}$ have unclear physical meaning and mode decomposition in terms of $G_{\mathrm{I}}$ and $G_{\mathrm{II}}$ can only be defined for a chosen finite crack extension $\Delta$.

The total strain energy release rate of an interface crack is well defined for an infinitesimal crack extension and agrees with Eq. 12 (Sun and Jih 1987; Raju et al. 1988; Dattaguru et al. 1994). The total strain energy release rate is obtained as follows:

$$
\begin{aligned}
G=G_{\mathrm{I}}+G_{\mathrm{II}}= & \operatorname{Lim}_{\Delta \rightarrow 0} \frac{1}{2 \Delta} \int_{0}^{\Delta} \sigma_{y y}(\Delta-r) \delta_{y}(r) \\
& +\sigma_{x y}(\Delta-r) \delta_{x}(r) \mathrm{d} r .
\end{aligned}
$$

The dependence of the SERR's components on the crack extension size was first presented in explicit mathematical form by Sun and Jih (1987) and Raju et al. (1988). The SERRs for an interface crack oscillate with $\Delta$ due to the oscillatory nature of stress and displacement fields. The oscillatory character of SERRs is similar to that of Eq. 3 , with the wavelength depending on the bimaterial parameter $\varepsilon$.
3.2 Analytical expressions for SERRs in terms of $K_{\mathrm{I}}$ and $K_{\mathrm{II}}$

The explicit mathematical form of SERR dependence on $\Delta$ has been analyzed, resulting in various suggested procedures to extract meaningful $\Delta$-independent parameters (Toya 1992; Chow and Atluri 1995; Beuth 1996; Sun and Qian 1997). In what follows, we will derive the analytical expressions of $G_{I}$ and $G_{I I}$ in terms of the complex stress intensity factors $K_{\mathrm{I}}$ and $K_{\mathrm{II}}$ and will show that seemingly different representations of SERRs are indeed the same.

The definition of SERRs in Eqs. 15 and 16 involves the product of stresses ahead of the crack tip and the displacement jumps between the crack faces behind the crack tip. From Eq. 7, the asymptotic stress field is given by

$$
\begin{aligned}
\sigma_{y y}= & \frac{1}{\sqrt{2 \pi r}}\left[K_{\mathrm{I}} \cos \left\{\varepsilon \ln \left(\frac{r}{l}\right)\right\}\right. \\
& \left.-K_{\mathrm{II}} \sin \left\{\varepsilon \ln \left(\frac{r}{l}\right)\right\}\right], \\
\sigma_{x y}= & \frac{1}{\sqrt{2 \pi r}}\left[K_{\mathrm{II}} \cos \left\{\varepsilon \ln \left(\frac{r}{l}\right)\right\}\right. \\
& \left.+K_{\mathrm{I}} \sin \left\{\varepsilon \ln \left(\frac{r}{l}\right)\right\}\right] .
\end{aligned}
$$

The expression for asymptotic displacements behind the crack tip in Eq. 10 can be reexpressed as:

$$
\begin{aligned}
& \delta_{y}+i \delta_{x}= m \sqrt{r}\left[\left(K_{\mathrm{I}}+2 \varepsilon K_{\mathrm{II}}\right)\right. \\
&\left.+i\left(K_{\mathrm{II}}-2 \varepsilon K_{\mathrm{I}}\right)\right]\left(\frac{r}{l}\right)^{i \varepsilon}, \\
& m=\frac{8}{\sqrt{2 \pi} E^{*}\left(1+4 \varepsilon^{2}\right) \cos (\pi \varepsilon)}
\end{aligned}
$$

From Eq. 21 the displacement field is:

$$
\begin{aligned}
\delta_{y}= & m \sqrt{r}\left[\left(K_{\mathrm{I}}+2 \varepsilon K_{\mathrm{II}}\right) \cos \left\{\varepsilon \ln \left(\frac{r}{l}\right)\right\}\right. \\
& \left.-\left(K_{\mathrm{II}}-2 \varepsilon K_{\mathrm{I}}\right) \sin \left\{\varepsilon \ln \left(\frac{r}{l}\right)\right\}\right], \\
\delta_{x}= & m \sqrt{r}\left[\left(K_{\mathrm{I}}+2 \varepsilon K_{\mathrm{II}}\right) \sin \left\{\varepsilon \ln \left(\frac{r}{l}\right)\right\}\right. \\
& \left.+\left(K_{\mathrm{II}}-2 \varepsilon K_{\mathrm{I}}\right) \cos \left\{\varepsilon \ln \left(\frac{r}{l}\right)\right\}\right] .
\end{aligned}
$$

Introducing Eqs. 20 and 22 into Eqs. 15 and 16, we obtain the expression for $G_{\mathrm{I}}$ and $G_{\mathrm{II}}$ used by Toya (1992)

$$
G_{\mathrm{I}}=\frac{m}{4 \sqrt{2 \pi}}\left\{f_{c} I_{c}-f_{s} I_{s}+\left(K_{I}^{2}+K_{\mathrm{II}}^{2}\right) I_{o}\right\},
$$




$$
G_{\mathrm{II}}=\frac{m}{4 \sqrt{2 \pi}}\left\{-f_{c} I_{c}+f_{s} I_{s}+\left(K_{\mathrm{I}}^{2}+K_{\mathrm{II}}^{2}\right) I_{o}\right\},
$$

where

$$
\begin{aligned}
& \left.I_{c}=\frac{1_{0}^{\Delta}}{a_{0}} \cos \varepsilon \ln \left(\frac{r(\Delta-r)}{l^{2}}\right)\right] \sqrt{\frac{r}{\Delta-r}} \mathrm{~d} r \\
& \left.I_{S}=\frac{1}{\Delta}{ }_{0}^{\Delta} \sin \varepsilon \ln \left(\frac{r(\Delta-r)}{l^{2}}\right)\right] \sqrt{\frac{r}{\Delta-r}} \mathrm{~d} r
\end{aligned}
$$

$$
\begin{aligned}
I_{0}= & \frac{1}{\Delta}{ }_{0}^{\Delta}\left\{\cos \varepsilon \ln \left(\frac{\Delta-r}{r}\right)\right] \\
& \left.\left.-2 \varepsilon \sin \varepsilon \ln \left(\frac{\Delta-r}{r}\right)\right]\right\} \sqrt{\frac{r}{\Delta-r}} \mathrm{~d} r
\end{aligned}
$$

$f_{c}=K_{\mathrm{I}}^{2}-K_{\mathrm{II}}^{2}+4 \varepsilon K_{\mathrm{I}} K_{\mathrm{II}}$,

$f_{s}=2\left[K_{\mathrm{I}} K_{\mathrm{II}}+\varepsilon\left(K_{\mathrm{II}}^{2}-K_{\mathrm{I}}^{2}\right)\right]$.

$(26 a, b)$

In addition to Eqs. 23 and 24 obtained by Toya (1992), other representations of $G_{\mathrm{I}}$ and $G_{\mathrm{II}}$ are available (Chow and Atluri 1995; Beuth 1996; Sun and Qian 1997). The representations involve complex quantities written in different forms; thus, it is not obvious that the expressions are related. However, here we will show that these representations can be derived from the expressions of Eqs. 23 and 24 and that they indeed are identical. We introduce two complex integrals, $A_{1}$ and $A_{2}$, to establish a connection between the various representations of $G_{\text {I }}$ and $G_{\text {II }}$. Let

$A_{1}=\frac{1}{\Delta}{ }_{0}^{\Delta}\left(\frac{r(\Delta-r)}{l^{2}}\right)^{i \varepsilon} \sqrt{\frac{r}{\Delta-r}} \mathrm{~d} r$

which can be rewritten as

$$
\begin{aligned}
A_{1} & =2\left(\frac{\Delta}{l}\right)_{0}^{2 i \varepsilon}\left(\frac{t}{t^{2}+1}\right)^{2(1+i \varepsilon)} \mathrm{d} t \\
& =2\left(\frac{\Delta}{l}\right)_{0}^{2 i \varepsilon} \sin ^{2} \theta(\sin \theta \cos \theta)^{2 i \varepsilon} \mathrm{d} \theta,
\end{aligned}
$$

where $t$ and $\theta$ are dummy variables. $A_{1}$ can be evaluated numerically (see Sects. 5.1.1 and 5.2.1) and be expressed as

$A_{1}=2\left(\frac{\Delta}{l}\right)^{2 i \varepsilon}|\Omega| e^{i \zeta}$,

where $|\Omega|$ and $\zeta$ depend only on the bimaterial parameter $\varepsilon$, and are obtained from the numerical integration. Also, let

$A_{2}=\frac{1}{\Delta}{ }_{0}^{\Delta}\left(\frac{\Delta-r}{r}\right)^{i \varepsilon} \sqrt{\frac{r}{\Delta-r}} \mathrm{~d} r$,

which can be expressed as

$$
\begin{aligned}
A_{2} & =2{ }_{0}^{\infty}\left(\frac{1}{t}\right)^{2 i \varepsilon}\left(\frac{t}{t^{2}+1}\right)^{2} \mathrm{~d} t \\
& =2{ }_{0}^{\pi / 2} \sin ^{2} \theta\left(\frac{\cos \theta}{\sin \theta}\right)^{2 i \varepsilon} \mathrm{d} \theta .
\end{aligned}
$$

We can relate the integrals $A_{1}$ and $A_{2}$ defined in Eqs. 27a and 28a, respectively, to the quantities used by Toya (1992) in Eq. 25 as:

$I_{c}=\operatorname{Re}\left\{A_{1}\right\}$,

$I_{S}=\operatorname{Im}\left\{A_{1}\right\}$,

$I_{0}=\operatorname{Re}\left\{A_{2}\right\}-2 \varepsilon \operatorname{Im}\left\{A_{2}\right\}$.

Furthermore, Eq. 26 can be re-expressed as

$f_{c}+i f_{s}=(1-2 i \varepsilon)\left(K_{\mathrm{I}}+i K_{\mathrm{II}}\right)^{2}$.

Substituting Eqs. 29 and 30 into Eqs. 23 and 24 obtained by Toya (1992), we re-express $G_{\mathrm{I}}$ and $G_{\mathrm{II}}$ as:

$$
\begin{aligned}
G_{\mathrm{I}}= & \frac{m}{4 \sqrt{2 \pi}} \operatorname{Re}\left\{\left[(1-2 i \varepsilon)\left(K_{\mathrm{I}}+i K_{\mathrm{II}}\right)^{2}\right] A_{1}\right. \\
& \left.+\left[K_{\mathrm{I}}^{2}+K_{\mathrm{II}}^{2}\right](1+2 i \varepsilon) A_{2}\right\}, \\
G_{\mathrm{II}}= & \frac{m}{4 \sqrt{2 \pi}} \operatorname{Re}\left\{-\left[(1-2 i \varepsilon)\left(K_{\mathrm{I}}+i K_{\mathrm{II}}\right)^{2}\right] A_{1}\right. \\
& \left.+\left[K_{\mathrm{I}}^{2}+K_{\mathrm{II}}^{2}\right](1+2 i \varepsilon) A_{2}\right\} .
\end{aligned}
$$

Equations 31 and 32 have been used by Chow and Atluri (1995) with the complex integral $A_{1}$ represented in explicit $\Delta$-dependent form given by Eq. 27b. Thus, we have shown that the expression used by Toya (1992) and Chow and Atluri (1995) are indeed the same equations. In addition, integral $A_{1}$ can be expressed in terms of a Gamma function or a Beta function (Magnus et al. 1966) 
and can be evaluated using standard mathematical tables. Sun and Jih (1987) and Sun and Qian (1997) have used integral $A_{1}$ expressed as a Gamma function and have obtained expressions for $G_{\mathrm{I}}$ and $G_{\mathrm{II}}$ similar to Eqs. 31 and 32.

Lastly, let us introduce the two real quantities $\Phi_{1}$ and $\Phi_{2}$ used by Beuth (1996):

$$
\begin{aligned}
\Phi_{1}= & \frac{1}{4 \Delta} \operatorname{Re}\left\{{ } _ { 0 } ^ { \Delta } \left(\sigma_{y y}(\Delta-r)\right.\right. \\
& \left.\left.+i \sigma_{x y}(\Delta-r)\right)\left(\delta_{y}(r)+i \delta_{x}(r)\right) \mathrm{d} r\right\}, \\
\Phi_{2}= & \frac{1}{4 \Delta} \operatorname{Re}\left\{\sigma _ { 0 } ^ { \Delta } \left(\sigma_{y y}(\Delta-r)\right.\right. \\
& \left.\left.+i \sigma_{x y}(\Delta-r)\right) \overline{\left(\delta_{y}(r)+i \delta_{x}(r)\right)} \mathrm{d} r\right\},
\end{aligned}
$$

where $\bar{z}$ denotes the complex conjugate of a complex number $z$. Substituting Eqs. 7, 10, and 27a into Eq. 33, and Eqs. 7, 10, and 28a into Eq. 34, we get

$$
\begin{aligned}
& \Phi_{1}=\frac{m}{4 \sqrt{2 \pi}} \operatorname{Re}\left\{(1-2 i \varepsilon)\left(K_{\mathrm{I}}+i K_{\mathrm{II}}\right)^{2} A_{1}\right\} ; \\
& \Phi_{2}=\frac{m}{4 \sqrt{2 \pi}}\left[K_{\mathrm{I}}^{2}+K_{\mathrm{II}}^{2}\right] \operatorname{Re}\left\{(1+2 i \varepsilon) A_{2}\right\} .
\end{aligned}
$$

Substituting Eqs. 35 and 36 into Eqs. 31 and 32, we obtain the expression used by Beuth (1996) as:

$G_{\mathrm{I}}=\Phi_{1}+\Phi_{2}$,

$G_{\mathrm{II}}=-\Phi_{1}+\Phi_{2}$

Thus, the expressions for $G_{\mathrm{I}}$ and $G_{\mathrm{II}}$ used in different approaches (Toya 1992; Chow and Atluri 1995; Beuth 1996; Sun and Qian 1997) are identical and can be derived from one another. All the available approaches can be used to characterize the interface crack by extracting meaningful $\Delta$-independent parameters. These $\Delta$-independent crack characterizing parameters are the $\Delta$-independent SERR-based mode mixity (Beuth 1996), or the SIF-based mode mixity (Toya 1992; Chow and Atluri 1995; Sun and Qian 1997), and shall be discussed in Chapter 4.
3.3 Implications of $\Phi_{1}$ and $\Phi_{2}$ with regard to $\Delta$-Dependence of $G_{\mathrm{I}}$ and $G_{\mathrm{II}}$

From Eq. 32 we have

$G=G_{\mathrm{I}}+G_{\mathrm{II}}=2 \Phi_{2}$.

Thus, $\Phi_{2}$ is half of the total strain energy release rate and is well defined. Hence, from Eq. 36, the complex integral $A_{2}$ is well defined. (It is also evident from Eq. 28b that $A_{2}$ shows no dependence on crack extension size $\Delta$ ). Furthermore, using Eqs. $12,29 \mathrm{c}, 36$, and 38 , the complex integral $I_{0}$ can be expressed as

$I_{0}=\frac{\pi\left(1+4 \varepsilon^{2}\right)}{2 \cosh (\pi \varepsilon)}$.

The quantity $\Phi_{1}$ can be related to $A_{1}$ from Eq. 35 and expanded using Eq. 27b, which shows clear dependence on the crack extension size $\Delta$. Hence, it is evident that the quantity $\Phi_{1}$ is responsible for the non-convergence of the components of the SERR. Individual SERRs in Eq. 37 can be re-expressed in the form used by Sun and Jih (1987) as:

$G_{\mathrm{I}}=\frac{G}{2}+\Phi_{1}, \quad G_{\mathrm{II}}=\frac{G}{2}-\Phi_{1}$.

$(40 a, b)$

Caution must be exercised when interpreting Eq. 40. Contrary to what has been indicated by Sun and Jih (1987), the result does not imply equal partition of the total strain energy release rate close to the crack tip as $\Delta \rightarrow 0$ : The quantity $\Phi_{1}$ is non-trivial and cannot be neglected (Itou 1986). The mode mixity very close to the crack tip can be examined from the contact model (Comninou 1977) which is more realistic: for contact of crack faces, $\delta_{y}$ vanishes, implying that mode I contribution must vanish (Toya et al. 1997). Thus, in the contact region and its vicinity (when $\Delta \rightarrow 0$ ) pure mode II dominance is expected. This agrees with the finite element results of Dattaguru et al. (1994) and Hemanth et al. (2005).

\subsection{Coupled strain energy release rate}

For a crack in a bimaterial interface, there is coupling between $\sigma_{y y}(\Delta-r)$ and $\delta_{x}(r)$, and between $\sigma_{x y}(\Delta-r)$ and $\delta_{y}(r)$. As a result, a coupled strain energy release rate can be introduced as suggested 
by Chow and Atluri (1995)

$$
\begin{aligned}
G_{\text {I-II }}= & \frac{1}{2 \Delta}{ }_{0}^{\Delta}\left[\sigma_{y y}(\Delta-r) \delta_{x}(r)\right. \\
& \left.+\sigma_{x y}(\Delta-r) \delta_{y}(r)\right] \mathrm{d} r=2 \Phi_{3},
\end{aligned}
$$

where after substituting Eqs. 7, 10 and $27 \mathrm{a}, \Phi_{3}$ can be expressed as

$\Phi_{3}=\frac{m}{4 \sqrt{2 \pi}} \operatorname{Im}\left\{(1-2 i \varepsilon)\left(K_{\mathrm{I}}+i K_{\mathrm{II}}\right)^{2} A_{1}\right\}$.

The expressions for $\Phi_{3}$ will be useful when rewriting the expressions for $\Delta$-independent parameters discussed in Chapter 4.

\subsection{Evaluation of $\Phi_{1}, \Phi_{2}, \Phi_{3}$}

The quantities $\Phi_{1}, \Phi_{2}$ and $\Phi_{3}$ can be computed from finite element calculations. Let $\mathbf{F}=F_{y, c}+i F_{x, c}$ be the nodal force vector for node $c$ (i.e., the force required to hold node $c$ and $d$ together), and $\Lambda=$ $\delta_{y, c-d}+i \delta_{x, c-d}$ be the nodal displacement jump vector. Thus, we have from Eqs. 33 and 34

$\Phi_{1}=\frac{1}{4 \Delta} \operatorname{Re}\{\mathbf{F} \boldsymbol{\Lambda}\}$,

$\Phi_{2}=\frac{1}{4 \Delta} \operatorname{Re}\{\mathbf{F} \overline{\boldsymbol{\Lambda}}\}$.

Also from Eq. 41, we have

$\Phi_{3}=\frac{1}{4 \Delta} \operatorname{Im}\{\mathbf{F} \boldsymbol{\Lambda}\}$.

\section{Obtaining mode mixities for interface crack using VCCT}

We will now review and extend the techniques that are available to extract mode mixities for interfacial cracks based on the VCCT. Expressions for various mode mixity definitions will be introduced for: (i) SERR-based mode mixity, (ii) $\Delta$-independent SERR-based mode mixity, (iii) SIF-based mode mixity obtained from oscillating SERRs, and (iv) SIF-based mode mixity obtained from complex strain energy release rate.

\subsection{SERR-based mode mixity}

Mode decomposition of the total strain energy release rate has successfully been implemented for cracks in homogeneous isotropic materials (Rybicki and Kanninen 1977), where the mode mixity based on SERRs, $\psi_{G}$, can be expressed as:

$$
\tan ^{2} \psi_{G}=\frac{G_{\mathrm{II}}}{G_{\mathrm{I}}} .
$$

For homogeneous, isotropic, linear-elastic materials, the results of mode mixity based on SERR, Eq. 46, and those based on SIFs, Eq. 8, are identical. However, for an interface crack, the decomposed components of the strain energy release rate, hence the mode mixity, $\psi_{G}$, depend on the crack extension size, $\Delta$. Thus, $\psi_{G}$ is referred to as $\Delta$-dependent mode mixity. This is illustrated for the two benchmarked problem considered in Sects. 5.1.1 and 5.2.1. However, when $\varepsilon$ is small, the oscillatory character can be ignored (Xie et al. 2004, 2005, 2006).

\section{2 $\Delta$-independent SERR-based mode mixity}

A method to obtain $\Delta$-independent SERR-based mode mixity was introduced by Beuth (1996). In this case, Eq. 40 can be modified to obtain $\Delta$ independent values of SERRs by eliminating the $\Delta$-dependence of $\Phi_{1}$. Thus, Eq. 35 is modified as (Beuth 1996)

$$
\Phi_{1}^{\prime}=\frac{m}{4 \sqrt{2 \pi}} \operatorname{Re}\left\{(1-2 i \varepsilon)\left(K_{\mathrm{I}}+i K_{\mathrm{II}}\right)^{2} A_{1}\left(\frac{l_{G}}{\Delta}\right)^{2 i \varepsilon}\right\}
$$

where $\Phi_{1}^{\prime}$ is a $\Delta$-independent quantity and $l_{G}$ is an arbitrary parameter used to normalize $\Delta$. Since $l_{G}$ is of the dimension "length," we will refer to it as the "normalizing length." We note that even though the modification of Eq. 47 has no real physical justification, this "mathematical trick" will be shown to be quite useful when using VCCT, as discussed in Sect. 4.5.3 below and illustrated in Sect. 5. Utilizing the expression for $\Phi_{1}$ in Eq. $35, \Phi_{3}$ in Eq. 42 , and noting that $\Delta$-dependence in these equations is due to $\Delta$-dependence of $A_{1}$ shown in Eq. 27 b, we can rewrite the Eq. 47 as

$\Phi_{1}^{\prime}=\operatorname{Re}\left\{\left(\Phi_{1}+i \Phi_{3}\right)\left(\frac{l_{G}}{\Delta}\right)^{2 i \varepsilon}\right\}$

In finite element calculation, it is convenient to use Eq. 48 for obtaining $\Phi_{1}^{\prime}$, where $\Phi_{1}$ and $\Phi_{3}$ are 
obtained numerically using Eqs. 43 and 45. Modifying Eq. 40 , the $\Delta$-independent SERRs can be obtained as

$G_{\mathrm{I}}^{\prime}=\frac{G}{2}+\Phi_{1}^{\prime}, \quad$ and $G_{\mathrm{II}}^{\prime}=\frac{G}{2}-\Phi_{1}^{\prime}$.

We can now introduce the following definition

$$
\tan ^{2} \psi_{G}^{\prime}=\frac{G_{\mathrm{II}}^{\prime}}{G_{\mathrm{I}}^{\prime}},
$$

where $\psi_{G}^{\prime}$ is the $\Delta$-independent SERR-based mode mixity. The reference length $l$ associated with the complex $K$ representation employed for expressing $G_{\mathrm{I}}$ and $G_{\mathrm{II}}$ in Eqs. 31 and 32, or Eq. 40 [which is analogous to Eq. 49], is the same as for complex $K$ in Eq. 8. However, it is obvious that $\Delta$-independent SERR-based mode mixity $\psi_{G}^{\prime}$ in Eq. 50 is not the same as SIF-based mode mixity $\psi$ in Eq. 8. It may be emphasized that the parameter $l_{G}$ is arbitrarily used to normalize $\Delta$, and has no physical significance, as opposed to the reference length $l$, which has clear physical meaning. The use of $\psi_{G}^{\prime}$ is illustrated for the two benchmarked problem considered in Sects. 5.1.1 and 5.2.1. In Sect. 4.5 we will derive a relationship between $\psi_{G}^{\prime}$ and $\psi$.

\subsection{SIF-based mode mixity from oscillating SERRs}

The SIF-based mode mixity, Eq. 8, can be obtained using the expressions of (oscillating) the components of the SERR. Dividing Eq. 23 with Eq. 24 for any chosen crack increment $\Delta$, the following simple expression - from which $K_{\mathrm{I}}$ and $K_{\mathrm{II}}$ can be extracted-is obtained:

$\frac{I_{c}\left(\kappa^{2}-1+4 \varepsilon \kappa\right)-2 I_{s}\left(\kappa+\varepsilon-\varepsilon \kappa^{2}\right)+I_{0}\left(\kappa^{2}+1\right)}{-I_{c}\left(\kappa^{2}-1+4 \varepsilon \kappa\right)+2 I_{S}\left(\kappa+\varepsilon-\varepsilon \kappa^{2}\right)+I_{0}\left(\kappa^{2}+1\right)}=\frac{G_{\mathrm{I}}}{G_{\mathrm{II}}}$

where $\kappa=K_{\mathrm{I}} / K_{\mathrm{II}}$ and the right hand side is obtained using the VCCT. Using $g=G_{\mathrm{II}} / G_{\mathrm{I}}$, and rearranging Eq. 51, we obtain a quadratic algebraic equation in $\kappa$

$$
\begin{aligned}
& \kappa^{2}\left[\left(I_{c}+2 \varepsilon I_{S}\right)(1+g)+I_{0}(g-1)\right] \\
& \quad+\kappa\left[\left(4 \varepsilon I_{c}-2 I_{S}\right)(1+g)\right] \\
& \quad-\left[\left(I_{c}+2 \varepsilon I_{S}\right)(1+g)-I_{0}(g-1)\right]=0 .
\end{aligned}
$$

Mode mixity is obtained by rewriting Eq. 8a as $\psi=\tan ^{-1}(1 / \kappa)$. Out of the two values obtained when solving the quadratic Eq. 52, the correct $\kappa$ can be ascertained from inspection by discarding the one which gives an incompatible $\psi$. The crack face displacements data from finite elements analysis and Eqs. 10 and 11 provides an approximate value of $\psi$ (Smelser 1979).

Several authors (Toya 1992; Chow and Atluri 1995; Sun and Qian 1997) have successfully obtained $K_{\mathrm{I}}$ and $K_{\mathrm{II}}$ from the SERR's components using the VCCT. The representations of their equations for $G_{\mathrm{I}}$ and $G_{\mathrm{II}}$ to extract $K_{\mathrm{I}}$ and $K_{\mathrm{II}}$ are different from Eq. 51, and are also different from one another. However, as examined in Sect. 3.2, all of the above approaches are identical and can be derived from one another. Thus, $\kappa$ obtained by any of the past approaches in literature (Toya 1992; Chow and Atluri 1995; Sun and Qian 1997) or Eq. 51 are the same. We are note that Eq. 51 (which further reduces to Eq. 52) is much simpler to use. The results from Eq. 51 and the previously derived methods discussed in the above paragraph are numerically investigated in Sects. 5.1.6 and 5.2.6.

4.4 SIF-based mode mixity using complex strain energy release rate

We will now consider an approach for obtaining SIF-based mode mixity (as defined by Eq. 8) using the complex strain energy release rate. Bjerken and Persson (2001) have cleverly introduced the complex strain energy release rate as

$$
\begin{aligned}
\Pi= & \operatorname{Lim}_{\Delta \rightarrow 0} \frac{1}{2 \Delta}{ }_{0}^{\Delta}\left[\sigma_{y y}(\Delta-r)\right. \\
& \left.+i \sigma_{x y}(\Delta-r)\right]\left[\delta_{y}(r)+i \delta_{x}(r)\right] \mathrm{d} r
\end{aligned}
$$

Substituting Eqs. 7, 10 and 27a into Eq. 53, we get

$$
\Pi=\frac{m}{2 \sqrt{2 \pi}}\left\{(1-2 i \varepsilon)\left(K_{\mathrm{I}}+i K_{\mathrm{II}}\right)^{2} A_{1}\right\},
$$

Further using Eqs. 35 and 42 into Eq. 54, and then utilizing Eqs. 43 and 45, we get

$$
\begin{aligned}
& \frac{\Pi}{2}=\left[\Phi_{1}+i \Phi_{3}\right], \quad\left[\Phi_{1}+i \Phi_{3}\right]=\frac{1}{4 \Delta} F \Lambda, \\
& \text { and } \Pi=\frac{1}{2 \Delta}[F \Lambda]
\end{aligned}
$$

$(55 \mathrm{a}, \mathrm{b}, \mathrm{c})$

Based on finite element simulations, $\Pi$ can be obtained from Eq. 55c, where $\mathbf{F}$ and $\boldsymbol{\Lambda}$ are the nodal 
force and displacement jump vectors introduced in Sect. 3.5. Further, $\mathbf{F}$ and $\boldsymbol{\Lambda}$ can be re-expressed as:

$\mathbf{F}=|\mathbf{F}| e^{i \chi_{c}}, \quad$ and $\quad \boldsymbol{\Lambda}=|\boldsymbol{\Lambda}| e^{i \phi_{c-d}}$,

where $\chi_{c}$ and $\phi_{c-d}$ are the arguments of complex nodal force and displacement jump, respectively. We substitute Eq. 56 into Eq. 55c to re-express the complex strain energy release rate. In addition, we substitute the expression for $A_{1}$ from Eq. $27 \mathrm{c}$ and expand Eq. 54. Equating the arguments of complex quantities in Eqs. 54 and 55c, we obtain the expression for obtaining mode mixity used by Bjerken and Persson (2001):

$\chi_{c}+\phi_{c-d}=2 \psi-\tan ^{-1}(2 \varepsilon)+2 \varepsilon \ln \left(\frac{\Delta}{l}\right)+\zeta$.

We recall that $\zeta$ is obtained from FE-simulations according to Eq. 27. Equation 57 utilizes only the complex integral $A_{1}$ and gives a simple expression for SIF-based mode mixity without a need for the relatively lengthy calculation involved in Eq. 51 . Eqs. $55-57$ are utilized in the numerical examples in Sects. 5.1.1 and 5.2.1.

\subsection{Relationships between $\psi, \psi_{G}$ and $\psi_{G}^{\prime}$}

In this section, we will establish relationships between the three mode mixity angles defined above:

(i) the SIF-based mode mixity, $\psi$ [defined by Eq. 8], for a given reference length $l$;

(ii) the $\Delta$-dependent SERR-based mode mixity, $\psi_{G}$ [defined by Eq. 46], for a given crack extension size, $\Delta$;

(iii) the $\Delta$-independent SERR-based mode mixity, $\psi_{G}^{\prime}$ [defined by Eq. 50], for a given crack extension size, $\Delta$, and a given normalizing length, $l_{G}$.

In particular, we will develop a new relationship between $\psi_{G}^{\prime}$ and $\psi$ for a special choice of the normalizing length, $l_{G}$.

\subsubsection{Relationship between $\psi$, and $\psi_{G}$}

First, let us consider the SERR-based, $\Delta$-dependent, mode mixity $\psi_{G}$. Using Eq. 46, and substituting Eq. 56 into the VCCT result of Eqs. 17 and 18, we have

$\tan ^{2}\left(\psi_{G}\right)=\tan \left(\chi_{c}\right) \tan \left(\phi_{c-d}\right)$, where $\chi_{c}$ and $\phi_{c-d}$ are defined with Eq. 56. Both $\chi_{c}$ and $\phi_{c-d}$ are readily available from the finite element simulations and are generally of the same magnitude (as shown in the numerical examples in Sects. 5.1.1 and 5.2.1), leading to the opportunity to derive some useful (but approximate) relationships. Thus, by developing an approximation for the right hand side of Eq. 58, we can determine an approximation of $\psi_{G}$. To this end, rewrite the arguments in Eq. 58 as

$$
\begin{array}{r}
\chi_{c}=\frac{\chi_{c}+\phi_{c-d}}{2}+\frac{\chi_{c}-\phi_{c-d}}{2} \text { and } \\
\phi_{c-d}=\frac{\chi_{c}+\phi_{c-d}}{2}-\frac{\chi_{c}-\phi_{c-d}}{2} .
\end{array}
$$

The right hand side of Eq. 58 can now be expressed as

$$
\begin{aligned}
& \tan \left(\chi_{c}\right) \tan \left(\phi_{c-d}\right) \\
& =\frac{\tan ^{2}\left[\frac{1}{2}\left(\chi_{c}+\phi_{c-d}\right)\right]-\tan ^{2}\left[\frac{1}{2}\left(\chi_{c}-\phi_{c-d}\right)\right]}{1-\tan ^{2}\left[\frac{1}{2}\left(\chi_{c}+\phi_{c-d}\right)\right] \tan ^{2}\left[\frac{1}{2}\left(\chi_{c}-\phi_{c-d}\right)\right]} \\
& \approx \tan ^{2}\left[\frac{1}{2}\left(\chi_{c}+\phi_{c-d}\right)\right] .
\end{aligned}
$$

Substituting the approximation defined by Eq. 60 into Eq. 58 results in

$\psi_{G} \approx \frac{1}{2}\left(\phi_{c-d}+\chi_{c}\right)$.

Equation 60 holds if $\left(\chi_{c}-\phi_{c-d}\right)$ is "small," and angles $\chi_{c}$ and $\phi_{c-d}$ are not "close" to either $0^{\circ}$ or $90^{\circ}$, which together implies that $\tan ^{2}\left(\chi_{c}-\phi_{c-d}\right)$ can be neglected. For example, if $\chi_{c}$ and $\phi_{c-d}$ differ by $4^{\circ}$ (which is the maximum value expected for most cases), the error in Eq. 61 is less than $0.2^{\circ}$ for $10^{\circ}<\psi_{G}<80^{\circ}$. Moreover, the error is less than $1^{\circ}$ for $3^{\circ}<\psi_{G}<87^{\circ}$. For cases when $\psi_{G}$ is outside the desired range, a different crack extension size can be selected. Thus, for most problems, Eq. 58 decomposes into the useful approximation expressed by Eq. 61 .

It follows that, by substituting Eq. 61 into Eq. 57, the relationship between SERR-based and SIFbased mode mixity ( $\psi$ and $\psi_{G}$, respectively) for most applications is given by

$\psi_{G} \approx \psi+\varepsilon \ln \left(\frac{\Delta}{l}\right)+\frac{1}{2}\left(\zeta-\tan ^{-1}(2 \varepsilon)\right)$

where we recall that that $\zeta$ is a numerically obtained parameter from Eq. 27 that depends on the bimaterial constant $\varepsilon$. As may be expected, this 
relationship is dependent on the crack extension size, $\Delta$.

Mantic and Paris (2004) have examined the relationship between SERR-based and SIF-based mode mixity following the work of Toya (1992). Furthermore, possible usefulness of $\psi_{G}$ in characterizing interfacial fracture toughness for physical specimens has been explored (Mantic and Paris, 2004, Zhao 2005). However, for interfacial cracks SERR-based mode mixity is non-unique and depend on the crack extension size, $\Delta$. Thus, any physical interpretation of $G_{\mathrm{I}}, G_{\mathrm{II}}$ and $\psi_{G}$ has to be explained in terms of finite crack extension size $\Delta$ as suggested by Toya (1992).

\subsubsection{Relationship between $\psi_{G}$ and $\psi_{G}^{\prime}$}

The $\Delta$-independent mode mixity, $\psi_{G}^{\prime}$, can serve as a useful crack characterizing parameter (Beuth 1996; Sun and Qian 1997; Mantic and Paris 2004). Thus, obtaining an expression that relates the $\Delta$ independent mode mixity, $\psi_{G}^{\prime}$, to the SIF-based mode mixity, $\psi$, is valuable. This will be achieved by first relating the two SERR-based mode-mixites $\left(\psi_{G}\right.$ and $\psi_{G}^{\prime}$ ) and then utilizing Eq. 62.

Using Eqs. 38 and 49, we rewrite Eq. 50 as:

$$
\tan ^{2} \psi_{G}^{\prime}=\frac{1-\Phi_{1}^{\prime} / \Phi_{2}}{1+\Phi_{1}^{\prime} / \Phi_{2}} \text {. }
$$

Rewriting Eq. 44 by introducing $\Phi_{4}$, we have

$$
\frac{1}{4 \Delta} \mathbf{F} \overline{\boldsymbol{\Lambda}}=\Phi_{2}+i \Phi_{4},
$$

where

$\Phi_{4}=\frac{1}{4 \Delta} \operatorname{Im}\{\mathbf{F} \bar{\Lambda}\}$.

Now, from Eqs. 64a and 55b, we have

$$
\left|\Phi_{1}+i \Phi_{3}\right|=\left|\Phi_{2}+i \Phi_{4}\right| \text {. }
$$

Based on results from the finite element calculation, $\Phi_{4}$ is obtained from Eq. 64b. In Eq. 56, we note that when $\chi_{c}$ is "close" to $\phi_{c-d}$, it follows that $\Phi_{4}$ is negligibly small compared to $\Phi_{2}$, and we have

$$
\left|\Phi_{2}+i \Phi_{4}\right| \approx \Phi_{2}
$$

The approximation in Eq. 66 is more restrictive on the values of $\chi_{c}$ and $\phi_{c-d}$ than in Eq. 61. For example, if $\chi_{c}$ and $\phi_{c-d}$ differ by $2^{\circ}$, the error incurred in Eq. 66 is about $3.5 \%$.
Next, we introduce $\Phi_{3}^{\prime}$ to rewrite Eq. 48 as

$$
\begin{gathered}
\Phi_{1}^{\prime}+i \Phi_{3}^{\prime}=\left(\Phi_{1}+i \Phi_{3}\right)\left(\frac{l_{G}}{\Delta}\right)^{2 i \varepsilon} \\
\text { and }\left|\Phi_{1}^{\prime}+i \Phi_{3}^{\prime}\right|=\left|\Phi_{1}+i \Phi_{3}\right| .
\end{gathered}
$$

Therefore, using the approximation in Eq. 66, it follows from Eq. $67 \mathrm{~b}$ that

$$
\left|\Phi_{1}^{\prime}+i \Phi_{3}^{\prime}\right| \approx \Phi_{2} \text {. }
$$

Further, we introduce $\tan (p)=\Phi_{3}^{\prime} / \Phi_{1}^{\prime}$, which from Eq. $67 \mathrm{a}, \mathrm{b}, \mathrm{c}$ leads to $\cos (p) \approx \Phi_{1}^{\prime} / \Phi_{2}$. Thus, Eq. 63 can be rewritten as

$$
\tan ^{2} \psi_{G}^{\prime} \approx \frac{1-\cos (p)}{1+\cos (p)}=\tan ^{2}\left(\frac{p}{2}\right),
$$

leading to

$\psi_{G}^{\prime} \approx \frac{p}{2}$.

Similarly, we rewrite Eq. 46 using Eq. 37, and introduce $\tan (q)=\Phi_{3} / \Phi_{1}$ to obtain $\psi_{G} \approx(q / 2)$. Therefore, using the values of quantities $p$ and $q$ obtained above, from the arguments of complex quantities in Eq. 67a, we finally get a relationship between $\psi_{G}^{\prime}$ and $\psi_{G}$

$\psi_{G}^{\prime} \approx \psi_{G}+\varepsilon \ln \left(\frac{l_{G}}{\Delta}\right)$.

When $l_{G}=\Delta$ it follows that $\psi_{G}^{\prime}\left(l_{G}=\Delta\right)=\psi_{G}$, i.e., when the normalizing length is selected as the crack extension length, the $\Delta$-dependent mode mixity equals that of the $\Delta$-independent mode mixity. ${ }^{1}$ Even though introduction of the normalizing length is a useful strategy, this observation serves to highlight the non-physical nature of $l_{G}$. Nevertheless, from Eq. 69a, we can relate $\psi_{G}^{\prime}$ from one normalizing length to another as

$\psi_{G, 2}^{\prime}=\psi_{G, 1}^{\prime}+\varepsilon \ln \left(\frac{l_{G, 2}}{l_{G, 1}}\right)$,

where $l_{G, 1}$ and $l_{G, 2}$ are two normalizing lengths used to define the mode mixities, $\psi_{G, 1}^{\prime} \equiv \psi_{G}^{\prime}\left(l_{G, 1}\right)$ and $\psi_{G, 2}^{\prime} \equiv \psi_{G}^{\prime}\left(l_{G, 2}\right)$. We note that Eq. 69b, obtained for $\Delta$-independent SERR-based mode mixity, has a similar form to Eq. 9 b used for SIF-based mode mixity.

1 This also follows from Eq. 48 and comparing Eq. 49 to 40. 


\subsubsection{Relationship between $\psi$ and $\psi_{G}^{\prime}$}

More insight into various expressions for mode mixities is obtained by selecting the normalizing length to equal the reference length. When $l_{G}=l$, Eqs. 69a and 62 yields:

$\psi_{G}^{\prime}\left(l_{G}=l\right) \approx \psi(l)+\frac{1}{2}\left(\zeta-\tan ^{-1}(2 \varepsilon)\right)$.

Thus, when the normalizing length, $l_{G}$, equals the reference length, $l$, the $\Delta$-independent SERRbased mode mixity, $\psi_{G}^{\prime}$, and the SIF-based mode mixity, $\psi$, are merely shifted by a phase angle $\frac{1}{2}\left[\zeta-\tan ^{-1}(2 \varepsilon)\right]$, which only depends on the bimaterial constant $\varepsilon$.

Hence, we have shown that $\Delta$-independent SERR-based mode mixity can be related to the SIF-based mode mixity by setting the normalizing length equal to the reference length. Even though $\psi_{G}^{\prime}$ appears to be a mathematical quantity with no physical meaning (unlike $\psi$ ), we believe $\psi_{G}^{\prime}$ can be a useful parameter if care is taken in its interpretation. The relationship defined in Eq. 70 may be useful when interpreting results using VCCT, since the phase shift only depends on the bi-material constant $\varepsilon$. The numerical results illustrating this are presented in Sects. 5.1.4 and 5.2.4.

\section{Numerical examples}

Two examples of interface cracks between dissimilar isotropic elastic layers are considered for determining and comparing the mode mixity values from the expressions obtained in Sect. 4. The two benchmark problems considered are: (i) an interface crack in an infinite bimaterial plate subjected to a uniform normal stress (Fig. 3A); and (ii) a bilayer four-point flexure specimen with an interface crack (Fig. 4A). Finite element models are constructed using the commercially available program ABAQUS (2003). For each case, symmetry condition about the $y$-axis at the mid-section of the specimen is imposed to reduce the model size. Four-node constant strain quadrilateral elements (CPE4R) are used. The finite element models of the two specimens are shown in Figs. 3B and 4B, respectively. The mesh around the crack tip for the flexure specimen is also shown in Fig. 4B. This mesh is similar for the bimaterial plate but omitted for brevity. The near tip mesh is symmetric about the crack tip with respect to both $x$ and $y$ axes, Fig. 2. Various crack extension sizes, $\Delta$, are considered by changing the near tip mesh. For the finest mesh, the bimaterial plate model has 16,114 elements and the four point flexure specimen model has 21,771 elements. The SIF-based mode mixity values computed using the VCCT from Eqs. 51 and 57, are compared with analytical solutions and results from the crack face displacement method (Matos et al. 1989).

\subsection{Interface crack in infinite plate subject to uniform tension}

The infinite bimaterial plate with a small, isolated crack at the interface subjected to a uniform tensile stress normal to the crack (Fig. 3) has been studied extensively (e.g. Rice and Sih 1965; Sun and Jih 1987; Toya 1992; Chow and Atluri 1995; Sun and Qian 1997; Bjerken and Persson 2001) and is thus a suitable benchmark problem. Plane strain condition is assumed. In our model, the material properties are: $E_{1}=200 \mathrm{GPa}, E_{2}=5 \mathrm{GPa}$, and $\nu_{1}=v_{2}=0.25$. Thus, $\alpha=0.9512, \beta=0.3171$, and $\varepsilon=-0.10453$. The plate size is $400 \mathrm{~mm} \times 400 \mathrm{~mm}$ and the crack length is $2 a=10 \mathrm{~mm}$. The analytical solution (Rice and Sih 1965) requires the uniform remote stresses along the $x$-direction to be unequal, $\sigma_{x x(1)}^{\infty}=\sigma_{x x(2)}^{\infty}$, in order to maintain the continuity of longitudinal normal strain, $\varepsilon_{x x}$, across the interface. Thus, the longitudinal normal stress is discontinuous across the interface and we have (Rice and Sih 1965)

$$
\begin{aligned}
& \sigma_{x x(2)}^{\infty}=\frac{\overline{E_{2}}}{\overline{E_{1}}} \sigma_{x x(1)}^{\infty} \\
& \quad+\frac{\sigma_{y y}^{\infty}}{1-v_{2}^{2}} v_{2}\left(1+v_{2}\right)-\frac{E_{2}}{E_{1}} v_{1}\left(1+v_{1}\right)
\end{aligned}
$$

The remote loading is $\sigma_{y y}^{\infty}=100 \mathrm{MPa}$; $\sigma_{x x(1)}^{\infty}=0$, and $\sigma_{x x(2)}^{\infty}=32.5 \mathrm{MPa}$.

\subsubsection{Mode mixities obtained from the VCCT}

First, we compute the $\Delta$-dependent SERR-based mode mixity discussed in Sect. 4.1. Using the VCCT, the components of the SERR, $G_{I}$ and $G_{I I}$, are 

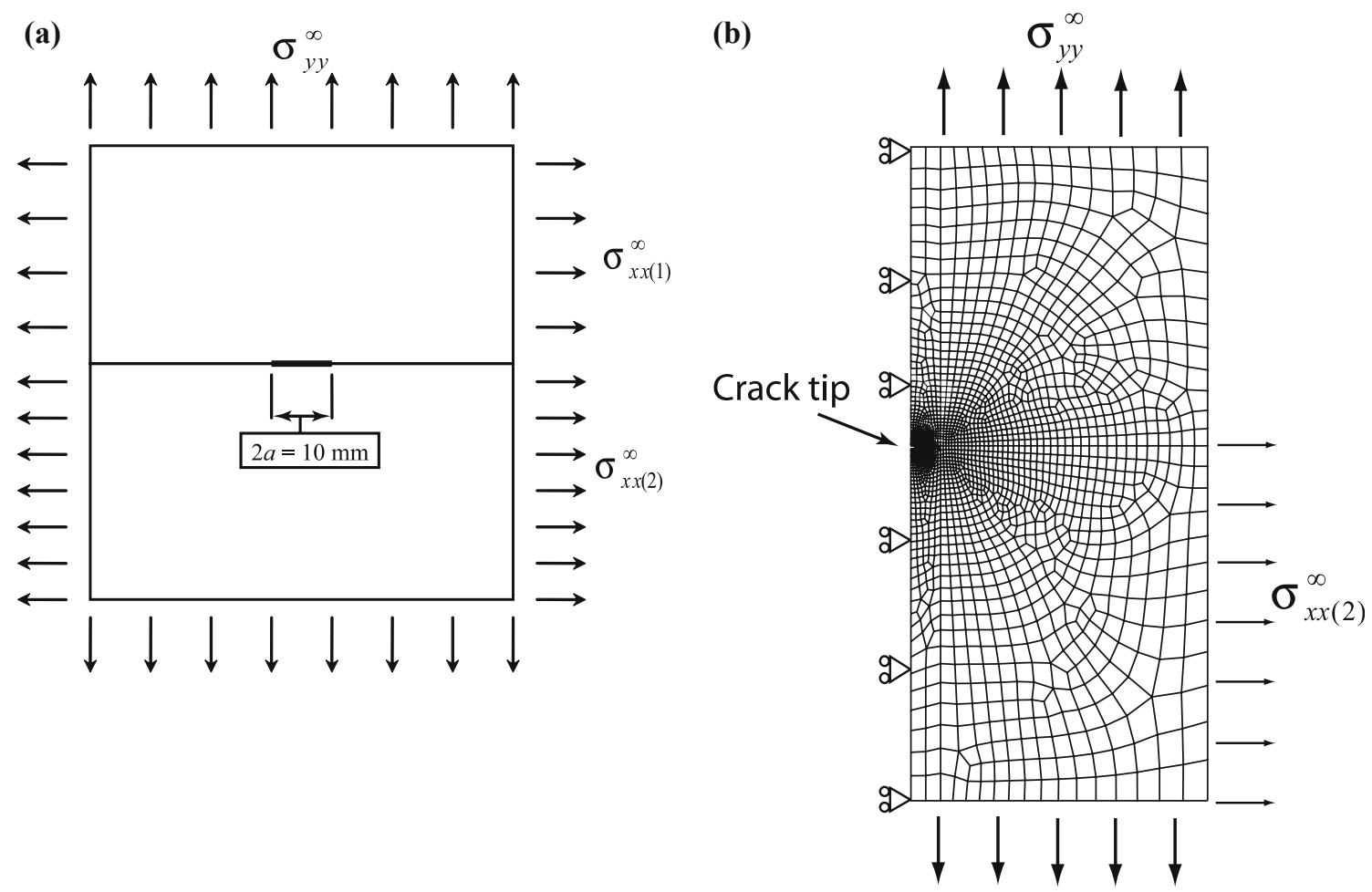

Fig. 3 (a) An interface crack in an infinite bimaterial plate, (b) the finite element mesh used for the simulations

computed numerically from Eqs. 17 and 18, and the SERR-based mode mixity, $\psi_{G}$, is obtained form Eq. 46. The total strain energy release rate, $G$, is independent of the crack extension size, but $G_{\mathrm{I}}$ and $G_{\mathrm{II}}$, thus $\psi_{G}$, depend on the crack extension size (Table 1, Fig. 5a). The average $G=$ 14.1547 $\mathrm{Nmm}^{-1}$.

Secondly, we compute the $\Delta$-independent SERR-based mode mixity introduced in Sect. 4.2. Equation 48 is used to determine the $\Delta$-independent quantity $\Phi_{1}^{\prime}$, and normalizing length $l_{G}$ is arbitrarily selected as $l_{G}=0.1 \mathrm{~mm}$. (The normalizing length is selected arbitrarily to provide positive values of $G_{\mathrm{I}}^{\prime}$ and $G_{\mathrm{II}}^{\prime}$ ) The $G$ value is taken as the average $G$ in Table 1 . The $\Delta$-independent SERRs are obtained using Eq. 49. From Eq. 50, we obtain consistent values of $\Delta$-independent SERRbased mode mixity, $\psi_{G}^{\prime}$. Similar results are obtained for all crack extension sizes as tabulated in Table 2 and shown in Fig. 5a. The average value is $\psi_{G}^{\prime}\left(l_{G}=0.1\right)=31.46^{\circ}$.

Next, we obtain the SIF-based mode mixity from the components of the SERR discussed in Sect. 4.3.
The complex quantity $A_{1}$ is obtained using numerical integration of Eq. 27b:

$$
\begin{aligned}
\left(\frac{l}{\Delta}\right)^{2 i \varepsilon} A_{1} \approx 1.48033+i 0.43716 & =1.54353\left(\arg 16.45^{\circ}\right) \\
& =1.54353 e^{0.2871 i},
\end{aligned}
$$

where $l=2 a=10 \mathrm{~mm}$ is the reference length. Equations 29a and 29b are used to obtain the quantities $I_{c}$ and $I_{s}$. Equation 39 yields $I_{0}=1.55486$. The mode mixity is obtained from Eq. 52 and results are tabulated in Table 3 . The results show some sensitivity with the crack extension size $\Delta$. For the finest near-tip mesh we have $\psi(l=10)=-10.30^{\circ}$ as shown in Fig. 5a.

Lastly, SIF-based mode mixity is alternatively obtained from the complex strain energy release rate as discussed in Sect. 4.4. We use the Eqs. 55 and 56 to obtain the complex strain energy release rate numerically, and the argument of complex quantity $A_{1}$ is available from numerical integration of Eq. $27 \mathrm{~b}$. The reference length, $l$, selected is the crack length $(10 \mathrm{~mm})$. The SIF-based mode mixity $\psi$ is 

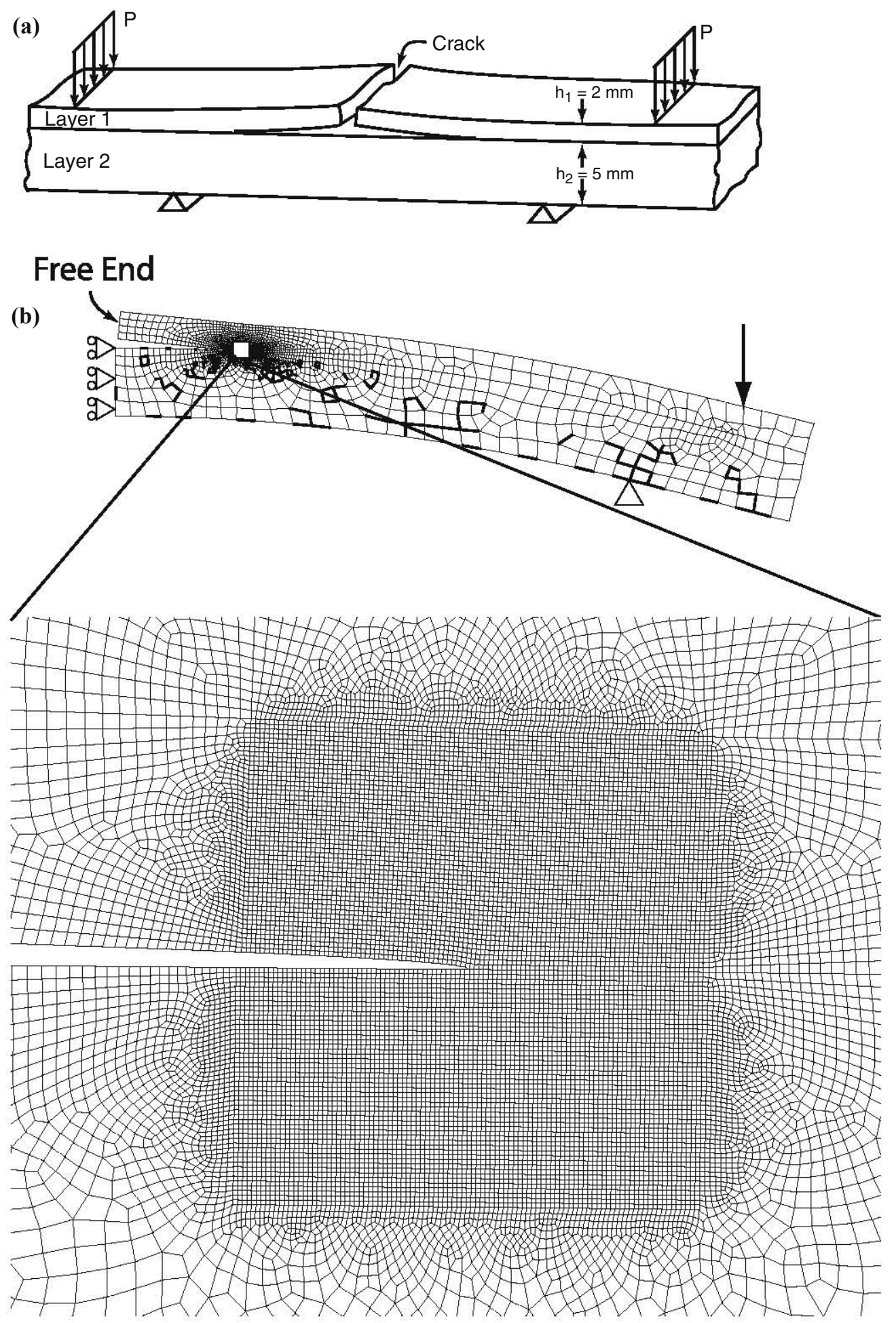

Fig. 4 (a) A four-point bend specimen with interfacial cracks, (b) the finite element mesh used for the simulations, including the near crack field 
Table 1 VCCT Results for SERRs for bimaterial plate

\begin{tabular}{|c|c|c|c|c|c|c|c|c|}
\hline $\begin{array}{l}\Delta \\
(\mathrm{mm})\end{array}$ & $\begin{array}{l}F_{y, c} \\
\left(\mathrm{Nmm}^{-1}\right)\end{array}$ & $\begin{array}{l}F_{x, c} \\
\left(\mathrm{Nmm}^{-1}\right)\end{array}$ & $\begin{array}{l}\delta_{y, c-d} 10^{-2} \\
(\mathrm{~mm})\end{array}$ & $\begin{array}{l}\delta_{x, c-d} 10^{-2} \\
(\mathrm{~mm})\end{array}$ & $\begin{array}{l}G_{\mathrm{I}} \\
\left(\mathrm{Nmm}^{-1}\right)\end{array}$ & $\begin{array}{l}G_{\mathrm{II}} \\
\left(\mathrm{Nmm}^{-1}\right)\end{array}$ & $\begin{array}{l}G \text { total } \\
\left(\mathrm{Nmm}^{-1}\right)\end{array}$ & $\begin{array}{l}\psi_{G} \\
(\operatorname{deg})\end{array}$ \\
\hline 0.1 & 54.3577 & 33.6988 & 3.7890 & 2.2877 & 10.2981 & 3.8546 & 14.1527 & 31.45 \\
\hline 0.05 & 36.6293 & 26.5310 & 2.5520 & 1.8089 & 9.3478 & 4.7992 & 14.1470 & 35.62 \\
\hline 0.02 & 21.4862 & 18.9194 & 1.4970 & 1.2933 & 8.0412 & 6.1171 & 14.1583 & 41.09 \\
\hline \multirow[t]{2}{*}{0.005} & 9.2793 & 10.9134 & 0.6460 & 0.7483 & 5.9944 & 8.1665 & 14.1609 & 49.41 \\
\hline & & & & & & Average & 14.1547 & \\
\hline
\end{tabular}

Fig. 5 Various definitions of mode mixities as a function of crack tip element size, $\Delta:(\mathbf{A})$ Bimaterial plate and (B) Four-point bend specimen. $\psi_{G}$ is the $\Delta$-dependent strain energy release rate based mode mixity; $\psi_{G}^{\prime}$ is the $\Delta$-independent strain energy release rate based mode mixity with normalizing length $l_{G}$; and $\psi$ is based on stress intensity factures with reference length $l$
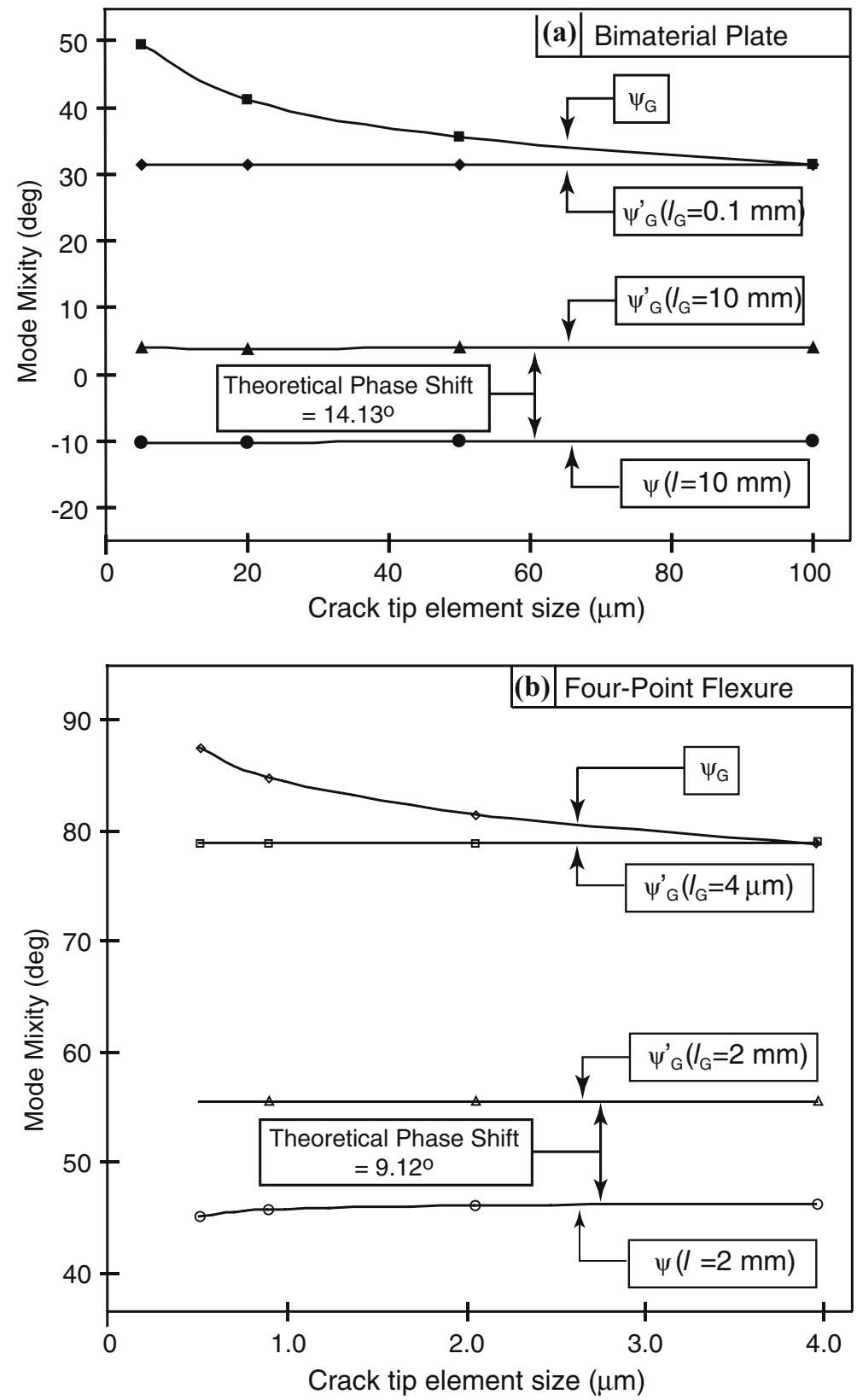
Table $2 \Delta$-independent SERR-based mode mixity $\psi_{G}^{\prime}$ for bimaterial plate

\begin{tabular}{llllllll}
\hline$\Delta$ & $\begin{array}{l}2 \varepsilon \ln \left(\frac{l_{G}}{\Delta}\right) \\
(\mathrm{mm})\end{array}$ & $\begin{array}{l}\Phi_{1} \\
(\mathrm{Neg})\end{array}$ & $\begin{array}{l}\Phi_{3} \\
\left(\mathrm{Nmm}^{-1}\right)\end{array}$ & $\begin{array}{l}\Phi_{1}^{\prime} \\
\left(\mathrm{Nmm}^{-1}\right)\end{array}$ & $\begin{array}{l}G_{I}^{\prime} \\
\left(\mathrm{Nmm}^{-1}\right)\end{array}$ & $\begin{array}{l}G_{\mathrm{II}}^{\prime} \\
\left(\mathrm{Nmm}^{-1}\right)\end{array}$ & $\begin{array}{l}\psi_{G}^{\prime}\left(l_{G}=0.1\right) \\
(\mathrm{deg})\end{array}$ \\
\hline 0.1 & 0.0 & 3.2217 & 6.3010 & 3.2217 & 10.2991 & 3.8556 & 31.46 \\
0.05 & -8.30 & 2.2743 & 6.6983 & 3.2174 & 10.2948 & 3.8600 & 31.48 \\
0.02 & -19.28 & 0.9620 & 7.0138 & 3.2239 & 10.3012 & 3.8534 & 31.45 \\
0.005 & -35.88 & -1.0860 & 6.9969 & 3.2209 & 10.2982 & 3.8564 & 31.46 \\
& & & & & & Average & 31.46 \\
\hline
\end{tabular}

Table 3 SIF-based mode mixity $\psi$ from the components of the SERR for bimaterial plate

\begin{tabular}{llllll}
\hline $\begin{array}{l}\Delta \\
(\mathrm{mm})\end{array}$ & $\begin{array}{l}2 \varepsilon \ln \left(\frac{\Delta}{l}\right) \\
(\mathrm{deg})\end{array}$ & $I_{c}$ & $I_{s}$ & $\kappa$ & $\begin{array}{l}\left(l_{=} 10\right) \\
(\mathrm{deg})\end{array}$ \\
\hline 0.1 & 55.16 & 0.48689 & 1.46472 & $-5.6439,-0.2986$ & -10.04 \\
0.05 & 63.46 & 0.27035 & 1.51967 & $-5.6128,-0.1473$ & -10.10 \\
0.02 & 74.44 & -0.02404 & 1.54334 & $-5.5571,0.0436$ & -10.20 \\
0.005 & 91.04 & -0.46396 & 1.47215 & $-5.5021,0.3443$ & -10.30 \\
\hline
\end{tabular}

obtained using Eq. 57. The results are similar for all cases and tabulated in Table 4 with the average value $\psi(l=10)=-10.25^{\circ}$.

\subsubsection{Mode mixity from the crack face displacement method}

For comparison, we will determine the mode mixity based on the near-tip displacement field available from Eq. 10a using the crack face displacement (CSD) method (Matos et al. 1989). This method extends the approach of Smelser (1979) to provide better accuracy of the mode mixity values. We square the modulus of complex terms on both sides of Eq. 10a and substitute Eq. 12 to obtain (Matos et al. 1989)

$\frac{\delta_{y}^{2}+\delta_{x}^{2}}{r}=64 \frac{G}{2 \pi\left(1+4 \varepsilon^{2}\right) E^{*}}$.
In Eq. 72, $G$ is obtained from Table 1. All other parameters in the right hand side are material properties, thus are known. The left hand side is obtained from finite element results of crack face displacement jumps at various distances from the crack tip, $r$. Although the left hand side should match with the right hand side for any $r$ in the $k$ annulus region, numerical error exists in the left hand side (Matos et al. 1989). We get best fit of the left hand side and the right hand side at $r=$ $0.35 \mathrm{~mm}$. (This value of $r$ does not have any physical meaning). Here, we have $\phi=20.53^{\circ}$, where $\phi$ is defined by Eq. 11. Equating the arguments of Eq. 10a on both sides, we get

$\phi=\psi+\tan ^{-1}(-2 \varepsilon)+\varepsilon \ln (r / l)$.

It follows that $\psi(l=10)=-11.36^{\circ}$, which is close to the mode mixity obtained by the VCCT calculations.

Table 4 SIF-based mode mixity $\psi$ from complex energy $\Pi$ for bimaterial plate

\begin{tabular}{lllll}
\hline$\Delta$ & $\begin{array}{l}\varepsilon \ln (\Delta / l) \\
(\mathrm{deg})\end{array}$ & $\begin{array}{l}\chi_{c} \\
(\mathrm{deg})\end{array}$ & $\begin{array}{l}\phi_{c-d} \\
(\mathrm{deg})\end{array}$ & $\begin{array}{l}\psi(l=10) \\
(\mathrm{deg})\end{array}$ \\
\hline 0.1 & 27.58 & 31.80 & 31.12 & -10.25 \\
0.05 & 31.73 & 35.92 & 35.33 & -10.24 \\
0.02 & 37.22 & 41.36 & 40.82 & -10.26 \\
0.005 & 45.52 & 49.63 & 49.20 & -10.24 \\
& & & Average & -10.25 \\
\hline
\end{tabular}




\subsubsection{Mode mixity from an analytical solution}

An alternative comparison is obtained from the analytical solution. For an isolated interface crack (length $=2 a$ ) in infinite bimaterial plate subject to remotely uniform stress $\sigma_{x y}^{\infty}, \sigma_{y y}^{\infty}$, the complex stress intensity factor is given by (Rice and Sih 1965; Rice 1988)

$K_{\mathrm{I}}+i K_{\mathrm{II}}=\left(\sigma_{12}^{\infty}+i \sigma_{22}^{\infty}\right) \sigma^{\infty} \sqrt{\pi a}(1+2 i \varepsilon)$,

where the complex stress intensity factor is based on the reference length $l=2 a$. This gives $\psi(l=10)$ $=\tan ^{-1}(2 \varepsilon)=-11.81^{\circ}$, again close to the values obtained by the VCCT.

\subsubsection{Phase shift between $\psi(l=10)$ and $\psi_{G}^{\prime}\left(l_{G}=10\right)$}

Using the SIF-based mode mixity results obtained from the oscillating SERRs in Sect. 5.1.1, we have $\psi(l=10)=-10.30^{\circ}$. Further, from the results for the $\Delta$-independent SERR-based mode mixity, and using Eq. $69 \mathrm{~b}$, we have $\psi_{G}^{\prime}\left(l_{G}=10\right)=3.88^{\circ}$. Thus, the phase shift, computed as $\left\{\psi_{G}^{\prime}\left(l_{G}=l\right)\right.$ $-\psi(l)\}$, is $14.18^{\circ}$, compared to the approximate value of $14.13^{\circ}$ from Eq. 70. The phase shift is illustrated in Fig. 5a.

\subsubsection{Size of the contact zone}

Finally, we investigate the size of the contact zone. For the interface crack in an infinite bimaterial plate, the analytical solution of the complex stress intensity factor is available from Eq. 74. Thus, the contact zone size given in Eq. 14 simplifies to

$r_{\mathrm{O}}=l \exp \left(\frac{\pi}{2 \varepsilon}\right)$.

For reference length $l=2 a=10 \mathrm{~mm}$, we find the contact zone size, $r_{\mathrm{O}}=2.98 \mathrm{~nm}$.

\subsubsection{Synopsis of results for the infinite plate}

Similar values for the SIF-based mode mixity were obtained from Eq. 51 [using oscillating SERRs] and using Eq. 57 [based on complex strain energy release rate]. Moreover, the crack face displacement method and the analytical solution both give similar values of the mode mixity (Table 5).
Figure 5A illustrates various mode mixities introduced in Sect. 4. Whereas $\psi_{G}$ depends on $\Delta$, the modified SERR-based mode mixity $\psi_{G}^{\prime}$ appears independent of $\Delta$. Letting the normalizing length equal the reference length, we have $\psi_{G}^{\prime}$ $\left(l_{G}=10\right)=3.88^{\circ}$. The SIF-based mode mixity is obtained form the Eq. 51, $\psi(l=10)=-10.30^{\circ}$. The phase shift $\left(14.18^{\circ}\right)$ between $\psi_{G}^{\prime}$ and $\psi$ when $l_{G} \equiv l$ is indicated in the Fig. 5A.

\subsection{Interface crack in four-point flexure specimen}

Next, consider a slender, bilayer four-point flexure specimen with an interface crack (Fig. 4). This specimen was designed by Charalambides et al. (1989) to measure interfacial fracture toughness of bilayer materials and has been studied extensively (e.g., Charalambides et al. 1989; Matos 1989; Hutchinson and Suo 1992; Suo and Hutchinson 1990). Analytical solutions for this specimen are available in form of total strain energy release rate and SIF-based mode mixity. Plane strain condition is assumed. The material properties selected for this benchmark problem are: $E_{1}=200 \mathrm{GPa}, E_{2}=20 \mathrm{GPa}$, and $v_{1}=v_{2}=0.33$. Thus, $\alpha=0.8182, \beta=0.2076$, and $\varepsilon=-0.067055$. The thickness of the upper layer is $h_{1}=2 \mathrm{~mm}$, and $h_{2}=5 \mathrm{~mm}$ for the bottom layer. The total length of the specimen is $100 \mathrm{~mm}$. The crack length is $9 \mathrm{~mm}$ on each side of the symmetry line. The distance between the inner supports is $74 \mathrm{~mm}$, and the outer loading points are separated by $90 \mathrm{~mm}$ with force $P=20 \mathrm{~N} / \mathrm{mm}$. Thus, a constant moment of $160 \mathrm{Nmm} / \mathrm{mm}$ is acting in the region between the inner supports (Fig. 4). Similar to Sect. 5.1 , various mode mixities defined in Chapter 4 will be obtained for this benchmark problem.

\subsubsection{Mode mixities obtained from the VCCT}

First, we compute the $\Delta$-dependent SERR-based mode mixity defined in Sect. 4.1. Using the VCCT, the components of the SERR, $G_{\mathrm{I}}$ and $G_{\mathrm{II}}$, are computed numerically from Eqs. 17 and 18, and the SERR-based mode mixity $\psi_{G}$ is obtained form Eq. 46. As for the infinite plate studied above and as expected, the total strain energy release rate, $G$, is independent of the crack extension size, but the 
Table 5 Comparison of mode mixity obtained from the four methods investigated ${ }^{\text {a }}$

\begin{tabular}{lll}
\hline & $\begin{array}{l}\text { Bimaterial plate specimen } \\
\varepsilon=-0.10453, l=10 \mathrm{~mm}\end{array}$ & $\begin{array}{l}\text { Four-point flexure specimen } \\
\varepsilon=-0.067055 l=2 \mathrm{~mm}\end{array}$ \\
\hline $\begin{array}{l}\text { Extracted from Oscillating } \\
\text { SERRs, Eq. 51 and Toya 1992; }\end{array}$ & -10.30 & 44.33 \\
Chow and Atluri 1995; Sun and & & \\
Qian 1997 & & 45.70 \\
$\begin{array}{l}\text { Based on complex strain energy } \\
\text { release rate Equation 57 by Bjer- }\end{array}$ & -10.25 & \\
ken and Persson (2001) & & 45.31 \\
$\begin{array}{l}\text { Crack face displacement method } \\
\text { (Matos et al. 1989) }\end{array}$ & -11.36 & 44.39 \\
$\begin{array}{l}\text { Analytical solution (Rice and Sih } \\
\text { 1965; Hutchinson and Suo 1990) }\end{array}$ & -11.81 & \\
\hline
\end{tabular}

a The representative value is taken as the value corresponding to the finest mesh when using the approach of oscillating SERRs, and the average value when using the complex strain energy release rate approach.

Table 6 VCCT results for SERRs for four-point flexure specimen

\begin{tabular}{|c|c|c|c|c|c|c|c|c|}
\hline $\begin{array}{l}\Delta \\
(\mathrm{mm})\end{array}$ & $\begin{array}{l}F_{y, c} \\
\left(\mathrm{Nmm}^{-1}\right)\end{array}$ & $\begin{array}{l}F_{x, c} \\
\left(\mathrm{Nmm}^{-1}\right)\end{array}$ & $\begin{array}{l}\delta_{y, c-d} \\
10^{-5}(\mathrm{~mm})\end{array}$ & $\begin{array}{l}\delta_{x, c-d} \\
10^{-5}(\mathrm{~mm})\end{array}$ & $\begin{array}{l}G_{\mathrm{I}} \\
10^{-4}\left(\mathrm{Nmm}^{-1}\right)\end{array}$ & $\begin{array}{l}G_{\mathrm{II}} \\
10^{-2}\left(\mathrm{Nmm}^{-1}\right)\end{array}$ & $\begin{array}{l}G \text { total } \\
10^{-2}\left(\mathrm{Nmm}^{-1}\right)\end{array}$ & $\begin{array}{l}\psi_{G} \\
(\operatorname{deg})\end{array}$ \\
\hline 0.004 & 0.274200 & 1.398500 & 5.100 & 25.580 & 17.4802 & 4.4717 & 4.6465 & 78.82 \\
\hline 0.002 & 0.149840 & 1.000100 & 2.700 & 18.180 & 10.1142 & 4.5454 & 4.6465 & 81.52 \\
\hline 0.0008 & 0.056397 & 0.638500 & 1.000 & 11.560 & 3.5248 & 4.6132 & 4.6484 & 85.00 \\
\hline 0.0004 & 0.019426 & 0.452915 & 0.300 & 8.190 & 0.7285 & 4.6367 & 4.6440 & 87.73 \\
\hline
\end{tabular}

components, $G_{\mathrm{I}}$ and $G_{\mathrm{II}}$, as well as $\psi_{G}$, depend on the crack extension size (Table 6, Fig. 5b). The average $G=4.6463\left(10^{-2}\right) \mathrm{Nmm}^{-1}$.

Secondly, we compute the $\Delta$-independent SERR-based mode mixity defined in Sect. 4.2. Equation 48 is used to determine the $\Delta$-independent quantity $\Phi_{1}^{\prime}$, with the normalizing length arbitrarily selected as $l_{G}=0.004 \mathrm{~mm}$. $G$ value is obtained from Table 6 . The $\Delta$-independent SERRs, $G_{\mathrm{I}}^{\prime}$ and $G_{\mathrm{II}}^{\prime}$, are obtained from Eq. 49. From Eq. 50 , we obtain consistent values of $\Delta$-independent SERR-based mode mixity, $\psi_{G}^{\prime}$. The $\Delta$-independent SERRs and $\Delta$-independent SERR-based mode mixity are tabulated in Table 7 . The results are similar for all cases, Fig. $5 \mathrm{~b}$, with the average value of $\psi_{G}^{\prime}\left(l_{G}=0.004\right)=78.84^{\circ}$.

Next, SIF-based mode mixity is obtained from the components of the SERR as discussed in Sect. 4.3. From numerical integration of Eq. $27 \mathrm{~b}$ we obtain

$$
\begin{aligned}
\left(\frac{l}{\Delta}\right)^{2 i \varepsilon} A_{1} & \approx 1.5327+i 0.2871=1.5594\left(\arg 10.61^{\circ}\right) \\
& =1.5594 e^{0.1852 i}
\end{aligned}
$$

where we set the reference length to be the thickness of the upper layer $l=h_{1}=2 \mathrm{~mm}$. Equations $29 \mathrm{a}$ and $29 \mathrm{~b}$ are used to obtain quantities $I_{c}$ and $I_{s}$. Equation 39 yields $I_{0}=1.5642$. The mode mixity is obtained from Eq. 52 and the results are tabulated in Table 8. For the smallest crack extension size, we get $\psi(l=2)=44.33^{\circ}$ as shown in Fig. 5b.

Lastly, SIF-based mode mixity is obtained using the complex strain energy release rate discussed in Sect. 4.4. We use Eqs. 55 and 56 to obtain the complex strain energy release rate numerically. The argument of complex quantity $A_{1}$ is available from numerical integration of Eq. 27b. The reference length $l$ selected is the thickness of upper layer $\left(h_{1}=2 \mathrm{~mm}\right)$. The SIF-based mode mixity $\psi$ is obtained using the Eq. 57. The mixity values are very close for all cases and tabulated in Table 9 with average value $\psi(l=2)=45.70^{\circ}$.

\subsubsection{Mode mixity from crack face displacement method}

As for the previous benchmark problem, we will compare the SIF-based mode mixity determined 
Table $7 \Delta$-independent SERR-based mode mixity $\psi_{G}^{\prime}$ for four-point flexure specimen

\begin{tabular}{llllllll}
\hline$\Delta$ & $2 \varepsilon \ln \left(\frac{l_{G}}{\Delta}\right)$ & $\Phi_{1}$ & $\Phi_{3}$ & $\Phi_{1}^{\prime}$ & $\begin{array}{l}G_{\mathrm{I}}^{\prime} \\
10^{-3}\left(\mathrm{Nmm}^{-1}\right)\end{array}$ & $\begin{array}{l}G_{\mathrm{II}}^{\prime} \\
10^{-2}\left(\mathrm{Nmm}^{-1}\right)\end{array}$ & $\begin{array}{l}\psi_{G}^{\prime}\left(l_{G}=0.004\right) \\
(\mathrm{deg})\end{array}$ \\
\hline 0.004 & 0.000 & -2.1484 & 8.8415 & -2.1484 & 1.7475 & 4.4716 & 78.82 \\
0.002 & -5.326 & -2.2222 & 6.7804 & -2.1497 & 1.7345 & 4.4728 & 78.86 \\
0.0008 & -12.367 & -2.2890 & 4.0326 & -2.1495 & 1.7365 & 4.4726 & 78.85 \\
0.0004 & -17.693 & -2.3147 & 1.8436 & -2.1492 & 1.7395 & 4.4724 & 78.84 \\
& & & & & & Average & 78.84 \\
\hline
\end{tabular}

Table 8 SIF-based mode mixity $\psi$ from the components of the SERR for four-point flexure specimen

\begin{tabular}{llllll}
\hline $\begin{array}{l}\Delta \\
(\mathrm{mm})\end{array}$ & $\begin{array}{l}2 \varepsilon \ln \left(\frac{\Delta}{l}\right) \\
(\mathrm{deg})\end{array}$ & $I_{c}$ & $I_{s}$ & $\kappa$ & $\begin{array}{l}\psi(l=2) \\
(\mathrm{deg})\end{array}$ \\
\hline 0.004 & 47.76 & 0.81779 & 1.32771 & $0.9856,0.3924$ & 45.42 \\
0.002 & 53.08 & 0.69116 & 1.39782 & $0.9888,0.5019$ & 45.32 \\
0.0008 & 60.12 & 0.51463 & 1.47199 & $1.0016,0.6574$ & 44.95 \\
0.0004 & 65.44 & 0.37594 & 1.51336 & $1.0237,0.7817$ & 44.33 \\
\hline
\end{tabular}

Table 9 SIF-based mode mixity $\psi$ from complex energy $\Pi$ for four-point flexure specimen

\begin{tabular}{lllll}
\hline $\begin{array}{l}\Delta \\
(\mathrm{mm})\end{array}$ & $\begin{array}{l}\varepsilon \ln \left(\frac{\Delta}{l}\right) \\
(\mathrm{deg})\end{array}$ & $\begin{array}{l}\chi_{c} \\
(\mathrm{deg})\end{array}$ & $\begin{array}{l}\phi_{c-d} \\
(\mathrm{deg})\end{array}$ & $\begin{array}{l}\psi(l=2) \\
(\mathrm{deg})\end{array}$ \\
\hline 0.004 & 23.88 & 78.91 & 78.72 & 45.67 \\
0.002 & 26.54 & 81.48 & 81.55 & 45.72 \\
0.0008 & 30.06 & 84.95 & 85.06 & 45.68 \\
0.0004 & 32.72 & 87.54 & 87.90 & 45.74 \\
& & & Average & 45.70 \\
\hline
\end{tabular}

from the VCCT calculations with values from the crack face displacement method, Eqs. 72 and 73. $G$ is obtained numerically from Table 6 . We get best fit of the left hand side and the right hand side for Eq. 72 at $r=0.002 \mathrm{~mm}$. Here, we have $\phi=79.49^{\circ}$. Thus $\psi(l=2)=45.31^{\circ}$.

\subsubsection{Mode mixity from the analytical solution}

The mode mixity can be obtained analytically using the local stress field near the crack tip. The solution for stress based mode mixity for a long, slender bimaterial beam with an interface crack subjected to mixed mode loading has been found using a combination of non-dimensional geometric parameters, bimaterial parameters, and a loading parameter to account for any general case (Hutchinson and Suo 1992; Suo and Hutchinson 1990). For the four-point flexure specimen (Fig. 4), we define $\eta=h_{1} / h_{2}$ as the thickness ratio of upper layer and bottom layer; $\Sigma=1 / \rho=E_{1}^{\prime} / E_{2}^{\prime}$ as the ratio of effective modulus; $1 / U=1+\Sigma \eta\left(4+6 \eta+3 \eta^{2}\right), 1 / V=$ $12\left(1+\Sigma \eta^{3}\right)$ and $\sin \gamma=\sqrt{U V}\left(6 \Sigma \eta^{2}(1+\eta)\right)$ as geometric parameters.

The mode mixity defined in Eq. 8a can be obtained in terms of $\omega$, a real angular quantity which depends only on $\eta, \alpha$ and $\beta$, and can be chosen in the range $0 \leq \omega \leq \pi / 2$. The dimensionless function $\omega(\eta, \alpha, \beta)$ can be obtained from tables (Suo and Hutchinson 1990). The mode mixity is obtained as $\tan \psi=\frac{\lambda \sin \omega-\cos (\omega+\gamma)}{\lambda \cos \omega+\sin (\omega+\gamma)}$ where
$\lambda=\frac{\bar{P} h_{1}}{\bar{M}} \sqrt{\frac{V}{U}}$.

In Eq. $76, \lambda$ is a load parameter, and $\bar{P}$ and $\bar{M}$ are the effective forces and moments, respectively 
(Hutchinson and Suo 1992; Suo and Hutchinson 1990). Here, $\eta=0.4 ; \Sigma=10 ; \omega=52.72$. Thus, $\bar{P}=33.90 \mathrm{~N} / \mathrm{mm}, \bar{M}=16.14 \mathrm{Nmm} / \mathrm{mm} ; 1 / U=$ $28.52,1 / V=19.68$; which gives $\gamma=34.56$ and $\lambda=5.057$. Therefore, we get $\psi(l=2)=\tan ^{-1}$ $(0.9789)=44.39^{\circ}$, which again is similar to the values obtained from the VCCT, above.

\subsubsection{Phase shift between $\psi(l=2)$ and $\psi_{G}^{\prime}\left(l_{G}=2\right)$}

Using the SIF-based mode mixity results obtained from the oscillating SERRs in Sect. 5.2.1, we have $\psi(l=2)=44.33^{\circ}$. Further, from the results for the $\Delta$-independent SERR-based mode mixity, and using Eq. 69b, we have $\psi_{G}^{\prime}\left(l_{G}=2\right)=54.96^{\circ}$. Thus, the phase shift, computed as $\left\{\psi_{G}^{\prime}\left(l_{G}=l\right)\right.$ $-\psi(l)\}$ is $10.63^{\circ}$, compared with approximate value of $9.12^{\circ}$ based on Eq. 70. The phase shift is illustrated in Fig. $5 b$.

\subsubsection{Size of the contact zone}

For an interface crack in the four-point flexure specimen, the contact zone can be obtained from Eq. 14. Using $\psi(l=2)=44.39^{\circ}$ from the analytical solution of Eq. 73 , we get $K_{\mathrm{II}}=0.9789$ $K_{\mathrm{I}}$. We obtain the size of the contact zone to be $r_{0}=1.91 \mathrm{~nm}$.

\subsubsection{Synopsis of results for the four-point flexure test}

As for the bimaterial plate, the values of the SIFbased mode mixity obtained from Eq. 51 [using oscillating SERRs] and those obtained using Eq. 57 [based on complex strain energy release rate] are similar. Moreover, the crack face displacement method and the analytical solution give similar results of the mode mixity (Table 5).

Figure 5B illustrates various mode mixities introduced in Sect. 4. Whereas $\psi_{G}$ depends on $\Delta$, the modified SERR-based mode mixity $\psi_{G}^{\prime}$ appears independent of $\Delta$. Setting the normalizing length equal to the reference length we have $\psi_{G}^{\prime}\left(l_{G}=2\right)=54.96^{\circ}$. The SIF-based mode mixity is obtained form the Eq. 51, $\psi(l=2)=44.33^{\circ}$.
The phase shift $\left(10.63^{\circ}\right)$ between $\psi_{G}^{\prime}$ and $\psi$ when $l_{G} \equiv l$ is indicated in Fig. 5B.

\section{Discussion}

\subsection{Accuracy of SIF-based mode mixity using VCCT}

SIF-based mode mixity results for two interface crack specimens - an infinite bimaterial plate and a four-point flexure specimen-were obtained in Sect. 5 using the VCCT. As summarized above, the values of the SIF-based mode mixity obtained from Eq. 51 using oscillating SERRs, are identical to the previous approaches (Toya 1992; Chow and Atluri 1995; Sun and Qian 1997). Furthermore, the SIFbased mode mixity values obtained using Eq. 57 based on the complex strain energy release rate (Bjerken and Persson 2001) were similar and independent of crack increment size, $\Delta$. The mode mixities obtained using these two methods were in addition compared to values based on the crack face displacement method and analytical solutions, and all methods were seen to give similar results (Table 5).

The accuracy of the mode mixity calculations will now be investigated through the complex stress intensity factors, $K_{\mathrm{I}}$ and $K_{\mathrm{II}}$. These can be obtained using Eq. 6, where the SIF-based mode mixity, $\psi$, is available from VCCT, and $|K|$ is obtained using the total strain energy release rate $G$, Eq. 12 . The error involved is computed as $\left(K_{\mathrm{I}}-K_{\mathrm{I}}^{\infty}\right) /|K|$ for $K_{\mathrm{I}}$ and as $\left(K_{\mathrm{II}}-K_{\mathrm{II}}^{\infty}\right) /|K|$ for $K_{\mathrm{II}}$, where $K_{\mathrm{I}}^{\infty}$ and $K_{\mathrm{II}}^{\infty}$ are the analytical solutions. All errors are less than $3 \%$, Table 10 . The errors of $K_{\mathrm{I}}$ and $K_{\mathrm{II}}$ obtain from the VCCT using Eqs. 51 and 57, are comparable to those reported previously (Matos et al. 1989; Chow and Atluri 1995; Sun and Qian 1997; Bjerken and Persson 2001).

For both specimens, the mode mixity values obtained using our Eqs. 51 and Eq. 57 by Bjerken and Persson (2001) are very close, with deviations of less than $1.5^{\circ}$ (Table 5). The method using Eq. 51 utilizes the expressions for oscillating SERRs in terms of complex quantities and is rigorous in mathematical details. The method using Eq. 57 utilizes complex strain energy release rate, is much simpler, and can provide reliable mode mixity values with an acceptable error. 
Table 10 Complex stress intensity factor and associated relative error for from the four methods investigated

\begin{tabular}{|c|c|c|c|c|}
\hline & \multicolumn{2}{|c|}{$\begin{array}{l}\text { Bimaterial plate specimen } \\
\beta=0.3171, E^{*}=5.2032 \mathrm{GPa} \\
G=14.1547 \mathrm{Nmm}^{-1},|K| \\
=9.0478 \mathrm{Nmm}^{-3 / 2}, l=10 \mathrm{~mm}\end{array}$} & \multicolumn{2}{|c|}{$\begin{array}{l}\text { Four-point flexure specimen } \\
\beta=0.2076, E^{*}=20.4038 \mathrm{GPa} \\
G=4.6463\left(10^{-2}\right) \mathrm{Nmm}^{-1},|K| \\
=0.9947 \mathrm{Nmm}^{-3 / 2}, l=2 \mathrm{~mm}\end{array}$} \\
\hline & $K_{\mathrm{I}}(\%$ error $)$ & $K_{\mathrm{II}}(\%$ error $)$ & $K_{\mathrm{I}}(\%$ error $)$ & $K_{\mathrm{II}}(\%$ error $)$ \\
\hline $\begin{array}{l}\text { Equation } 51 \text { based on VCCT } \\
\text { using oscillating SERRs }\end{array}$ & $8.9020(0.51 \%)$ & $-1.6178(2.59 \%)$ & $0.7115(0.07 \%)$ & $0.6951(-0.07 \%)$ \\
\hline $\begin{array}{l}\text { Equation } 57 \text { based on VCCT } \\
\text { using complex strain energy re- } \\
\text { lease rate }\end{array}$ & $8.9034(0.52 \%)$ & $-1.6100(2.67 \%)$ & $0.6947(-1.62 \%)$ & $0.7119(1.62 \%)$ \\
\hline $\begin{array}{l}\text { Crack Face Displacement } \\
\text { Method (Matos et al. 1989) }\end{array}$ & $8.8705(0.16 \%)$ & $-1.7822(0.77 \%)$ & $0.6995(-1.14 \%)$ & $0.7109(1.52 \%)$ \\
\hline $\begin{array}{l}\text { Analytical Solution (Rice and } \\
\text { Sih 1965; Hutchinson and Suo } \\
\text { 1990) }\end{array}$ & 8.8563 & -1.8518 & 0.7108 & 0.6958 \\
\hline
\end{tabular}

\subsection{Implications of contact zone}

The contact zone in the two benchmark problems considered in Chapter 5 are of the orders of nanometer, which is small compared to material length scale dimensions, e.g., the grain size. In general, the contact zone is very small. For example, for the bimaterial plate subjected to uniform tension and with an interfacial crack length of $2 a=10 \mathrm{~mm}$, the contact zone size is $6.04\left(10^{-71}\right) \mathrm{m}, 2.27\left(10^{-16}\right) \mathrm{m}$, $1.51\left(10^{-9}\right) \mathrm{m}, 2.83\left(10^{-7}\right) \mathrm{m}$ for $\varepsilon=-0.01,-0.05$, $-0.1,-0.15$, respectively [using Eq. 75]. This agrees with previous observations (e.g., Rice 1988; Sun and Qian 1997; Borovkov et al. 2000). Hence, the contact zone is much smaller than the $k$-annulus region, which is often scaled by the smallest dimension of the specimen or crack length (Wang and Suo 1990; Becker 1997). Thus, for moderate values of the bimaterial constant, the interfacial fracture mechanics solution, based on the $k$-annulus concept is reasonable. We note that for in-plane shear load, the contact zone size is larger than the values considered here, but remains small compared to other material dimensions.

Thus, the solution based on the "open crack model" (Williams 1959; Rice and Sih 1965) can be adopted to characterize interface crack singularity for moderate values of $\varepsilon$. However, due to the oscillatory nature of the solution, the open crack model is unable to infer the physical nature of (stressbased) mode mixity close to the crack tip, as a suitable reference length in the $k$-annulus region that governs the physical fracture process is not evident (Hutchinson and Suo 1992). However, (as noted in Sect. 3.3) according to the contact model (Comninou 1977), the crack grows in mode II inside the contact zone and its vicinity, although macroscopically the crack grows in mixed mode.

For material combinations with large mismatch vales, the contact zone can be larger than the atomic spacing, and the crack may grow in pure mode II for all cases of loading. Consequently, the fracture toughness of such bimaterial may be constant, as mode II is always expected to control the crack growth.

\section{Concluding remarks}

This paper reviews, unifies and extends methods characterizing interfacial fractures, with particular application on evaluating mode mixity using the finite element based virtual crack closure technique (VCCT).

It is well established that the complex stress intensity factors (SIFs), $K_{\mathrm{I}}$ and $K_{\mathrm{II}}$, can be used as the characterizing parameters for an interface crack singularity, i.e., the same SIFs in two different cracked bodies implies the same stress distribution close to the crack tip. The SIF-based mode mixity can be obtained from the oscillating components of the strain energy release rate (SERR) using the VCCT, either from our Eq. 51, or from past works (Toya 1992; Chow and Atluri 1995; Sun and Qian 
1997). We show that all of these approaches are identical and can be derived from one another. Our Eq. 51 is -in our opinion-significantly easier to use than the alternative methods referenced. Furthermore, we compare these methods to a different approach developed by Bjerken and Persson (2001) and show that this method gives acceptable values of mode mixity with significantly less computational efforts. Thus, either our Eq. 51 or the method presented by Bjerken and Persson (2001), as given in Eq. 57 may be the preferred methods to determine the SIF-based mode mixity from VCCT.

Since the VCCT directly yields a decomposed form of strain energy release rate, it would be convenient to use these two components directly to determine mode mixity. However, these components cannot directly be linked to mode I and mode II for an interface crack. Indeed, the components - and the SERR-based mode mixity - will depend on the element size at the crack tip, that is, to the crack extension size, $\Delta$. Thus, the dependence on element size is referred to as " $\Delta$-dependent SERR-based mode mixity." Evidently, this is not a useful design parameter. However, a " $\Delta$ independent SERR-based mode mixity" can be defined by introducing a "normalizing length parameter" (Beuth 1996). We show that when the reference length (used for the SIF-based mode mixity) and the normalizing length (used for $\Delta$ independent SERR-based mode mixity) are equal, the two mode mixities are only shifted by a phase angle, depending on the bimaterial parameter $\varepsilon$. Thus, even though $\Delta$-independent SERR-based mode mixity appears to be a mathematical quantity with no physical meaning, we believe it can be a useful parameter if care is taken in its interpretation.

Acknowledgements The authors would like to acknowledge that this work was supported by ONR-N00014-04-10498 .

\section{References}

ABAQUS (2003) Version 6.4, Hibbit, Karlsson, \& Sorenson Inc

Becker Jr TL, McNaney JM, Cannon RM, Ritchie RO (1997) Limitations on use of the mixed mode delaminating beam test specimen: effects of the size of the region of K-dominance. Mech of Mat 25:291-308
Beuth JL (1996) Separation of crack extension modes in orthotropic delamination models. Int J of Frac 77:305-321

Bjerken C, Persson C (2001) A numerical method for calculating stress intensity factors for interface cracks in bimaterials. Eng Frac Mech 68:235-246

Borovkov A, Palmov V, Banichuk N, Saurin V, Barthold F, Stein E (2000) Macro-failure criterion for the theory of laminated composite structures with free edge delaminations. Comp Str 76:195-204

Charalambides PG, Lund J, Evans AG, McMeeking RM (1989) A test specimen for determining the fracture resistance of bimaterial interfaces. J App Mech 56: 77-82

Cherepanov GP (1979) Mechanics of brittle fracture, Mc-Graw Hill

Chow TW, Atluri SN (1995) Finite element calculation of stress intensity factors for interfacial crack using virtual crack closure integral. Comput Mech 16:417-25

Comninou M (1977) The interface crack. J App Mech 1977:631-636

Dattaguru B, Venkatesha KS, Ramamurthy TS, Buchholz FG (1994) Finite element estimates of strain energy release rate components at the tip of an interface crack under mode I loading. Eng Frac Mech. 39(3): 451-463

Dudurs J (1969) Edge-bonded dissimilar orthogonal elastic wedge. J Appl Mech 36:650-652

England AH (1965) A crack between dissimilar media. J Appl Mech 32:400-402

Evans AG, Ruhle M, Dalgleish BJ, Charalambides PG (1990) The fracture energy of bimaterial interfaces. Mat Sc Eng A126:53-64

Hemnath D, Aradhya KSA, Ramamurthy TS, Raju NG (2005) Strain energy release rates for an interface crack in orthotropic media- finite element investigation. Eng Frac Mech 72:759-772

Hutchinson JW, Suo Z (1992) Mixed mode cracking in layered materials. Adv Appl Mech 29:63-89

Ikeda T, Miyazaki N, Soda T (1998) Mixed mode fracture criterion of interface crack between dissimilar materials. Eng Frac Mech 59(6):725-735

Irwin GR (1957) Analysis of stresses and strains near the end of a crack traversing a plate. J Appl Mech 24: 361-364

Itou S (1986) Stresses around an interface crack. Eng Frac Mech 25(4):415-420

Karlsson AM, Evans AG (2001) Numerical model for the cyclic instability of thermally grown oxides in thermal barrier systems. Acta Mater 49(10):1793-1804

Magnus W, Oberhettinger F, Soni RP (1966) Formulas and theorems for special functions of mathematical physics. Springer-Verlag, Berlin

Malyshev BM, and Salganik RL (1965) The strength of adhesive joints using the theory of cracks. Int J Frac 1: 114-128

Mantic V, Paris F (2004) Relation between SIF and ERR based measures of fracture mode mixity in interface cracks. Int J Frac 130:557-569

Matos PPL, McMeeking RM, Charalambides PG, Drory MD (1989) A method for calculating stress intensities in bimaterial interfaces. Int J Frac 40:235-254 
O'Dowd NP, Shih CF, Stout MG (1992) Test geometries for measuring interfacial fracture toughness. Int J Solids St 29(5):571-589

Raju IS, Crews JH, Aminpour MA (1988) Convergence of strain energy release rate components for edge delaminated composite materials. Eng Frac Mech 30:383-396

Parks DM (1974) A stiffness derivative finite element technique for determination of crack tip stress intensity factors. Int J Frac 10(4):487-502

Rice JR, Sih GC (1965) Plane problems of cracks in dissimilar media. J App Mech 32:418-423

Rice JR (1968) A path independent integral and the approximate analysis of strain concentration by notches and cracks. J Appl Mech 35:379-386

Rice J (1988) Elastic fracture mechanics concepts for interface cracks. J App Mech 55:98-103

Rice JR, Suo Z, Wang J-S (1990) Mechanics and thermodynamics of brittle interfacial failure in bimaterial systems, metal-ceramic interfaces. Ruhle M, Evans AG, Ashby MF, Hirth JP (eds). Acta-Scripta Metallurgica Proceedings Series 4, pp 269-294

Rybicki EF, Kanninen MF (1977) A finite element calculation of stress intensity factors by a modified crack closure integral. Eng Frac Mech 9:931-938

Smelser RE (1979) Evaluation of stress intensity factors for bimaterial bodies using numerical crack flank displacement data. Int J Frac 15(2):135-143

Sun CT, Jih CJ (1987) On strain energy release rates for interfacial cracks in bi-material media. Eng Frac Mech 28(1):13-20

Sun CT and Qian W (1997) The use of finite extension strain energy release rates in fracture of interfacial cracks. Int J Solids Str 34(20):2595-2609

Suo Z, Hutchinson JW (1990) Interface crack between two elastic layers. Int J Frac 43:1-18
Toya M (1992) On mode I and mode II energy release rates of interface crack. Int J Frac 56:345-352

Toya M, Aritomi M, Chosa A (1997) Energy release rates for an interface crack embedded in a laminated beam subjected to three-point bending. J Appl Mech 64: 375-382

Wang J-S, Suo Z (1990) Experimental determination of interfacial toughness curves using Brazil-nut sandwiches. Acta Mater 38(7):1279-1290

Westergaard HM (1939) Bearing pressure and cracks. Trans ASME 61:A49-A53

Williams ML (1959) The stresses around a fault or crack in dissimilar media. Bull Seismol Soc Am 49:199-204

Yau JF, Wang SS, Corten HT (1980) A mixed mode crack analysis of isotropic solids using conservation laws of energy. J Appl Mech 47:335-341

Xie D, Waas AM, Shahwan KW, Schroeder JA, Boeman RG (2004) Computation of strain energy release rate for kinking cracks based on virtual crack closure technique. CMES 6:515-524

Xie D, Waas AM, Shahwan KW Schroeder JA, Boeman RG (2005) Fracture criteria for kinking cracks in triple material bonded joints. Eng Fract Mech 72:2487-2504

Xie D, Chung J, Waas AM, Shahwan KW, Schroeder JA, Boeman RG, Kunc V, Klett LB (2006) Failure analysis of adhesively bonded structures: from coupon level data to structure level predictions and verification. Int J Fract 134:231-250

Yuuki R, Liu J-Q, Xu J-Q, Ohira T, Ono T (1994) Mixed mode fracture criteria for an interface crack. Eng Frac Mech 47(3):367-377

Zhao J-H (2005) Application of virtual crack closure integral method for interface cracks in low-k integrated circuit devices under thermal load. Eng Frac Mech 72:1361-1382 